\title{
Hydrogen cyanide and isocyanide in prestellar cores ${ }^{\star}$
}

\author{
M. Padovani ${ }^{1,2}$, C. M. Walmsley ${ }^{2,3}$, M. Tafalla ${ }^{4}$, P. Hily-Blant ${ }^{5}$, and G. Pineau des Forêts ${ }^{6,7}$ \\ ${ }^{1}$ Institut de Ciències de l'Espai (CSIC-IEEC), Campus UAB, Facultat de Ciències, Torre C5-parell 2a , 08193 Bellaterra, Spain \\ e-mail: padovani@ieec.uab.es \\ 2 INAF - Osservatorio Astrofisico di Arcetri, Largo E. Fermi 5, 50125 Firenze, Italy \\ 3 Dublin Institute of Advanced Studies, 31 Fitzwilliam Place, Dublin, Ireland \\ 4 Observatorio Astronómico Nacional (IGN), Alfonso XII 3, 28014 Madrid, Spain \\ 5 Université Joseph Fourier et CNRS, Institut de Planétologie et d'Astrophysique, 38041 Grenoble Cedex 9, France \\ ${ }^{6}$ IAS (UMR 8617), Université de Paris-Sud, 91405 Orsay, France \\ 7 LERMA (UMR 8112), Observatoire de Paris, 61 avenue de l'Observatoire, 75014 Paris, France
}

Received 25 April 2011 / Accepted 19 August 2011

\begin{abstract}
Aims. We studied the abundance of $\mathrm{HCN}, \mathrm{H}^{13} \mathrm{CN}$, and $\mathrm{HN}^{13} \mathrm{C}$ in a sample of prestellar cores, in order to search for species associated with high density gas.

Methods. We used the IRAM 30 m radiotelescope to observe along the major and the minor axes of L1498, L1521E, and TMC 2, three cores chosen on the basis of their $\mathrm{CO}$ depletion properties. We mapped the $J=1 \rightarrow 0$ transition of $\mathrm{HCN}, \mathrm{H}^{13} \mathrm{CN}$, and $\mathrm{HN}{ }^{13} \mathrm{C}$ towards the source sample plus the $J=1 \rightarrow 0$ transition of $\mathrm{N}_{2} \mathrm{H}^{+}$and the $J=2 \rightarrow 1$ transition of $\mathrm{C}^{18} \mathrm{O}$ in TMC 2 . We used two different radiative transfer codes, making use of recent collisional rate calculations, in order to determine more accurately the excitation temperature, leading to a more exact evaluation of the column densities and abundances.

Results. We find that the optical depths of both $\mathrm{H}^{13} \mathrm{CN}(1-0)$ and $\mathrm{HN}^{13} \mathrm{C}(1-0)$ are non-negligible, allowing us to estimate excitation temperatures for these transitions in many positions in the three sources. The observed excitation temperatures are consistent with recent computations of the collisional rates for these species and they correlate with hydrogen column density inferred from dust emission. We conclude that $\mathrm{HCN}$ and $\mathrm{HNC}$ are relatively abundant in the high density zone, $n\left(\mathrm{H}_{2}\right) \sim 10^{5} \mathrm{~cm}^{-3}$, where CO is depleted. The relative abundance $[\mathrm{HNC}] /[\mathrm{HCN}]$ differs from unity by at most $30 \%$ consistent with chemical expectations. The three hyperfine satellites of HCN(1-0) are optically thick in the regions mapped, but the profiles become increasingly skewed to the blue (L1498 and TMC 2) or red (L1521E) with increasing optical depth suggesting absorption by foreground layers.
\end{abstract}

Key words. ISM: abundances - ISM: molecules - ISM: clouds - ISM: general - radio lines: ISM - molecular data

\section{Introduction}

Tracing the dense central regions of prestellar cores is essential for an understanding of their kinematics and density distribution. Many of the well studied nearby cores are thought to be static with a "Bonnor-Ebert" radial density distribution (see Bergin \& Tafalla 2007, and references therein) comprising a roughly $1 / r^{2}$ fall off surrounding a central region of nearly constant density. However, our understanding of the central regions with $\mathrm{H}_{2}$ number densities of $10^{5} \mathrm{~cm}^{-3}$ or more is limited both by our uncertainties about dust emissivity and molecular abundances. Dust emission, because it is optically thin, is a useful tracer of mass distribution, but both temperature gradients and gradients in the dust characteristics (refractive index and size distribution) mean that dust emission maps should be interpreted with caution. Molecular lines offer the advantage that they permit an understanding of the kinematics, but depletion and other chemical effects render them often untrustworthy. Depletion is more rapid at high density and low temperature and these are just the effects that become important in the central regions of cores.

One interesting result from studies to date (e.g. Tafalla et al. 2006) is that the only molecular species to have a spatial

\footnotetext{
* Based on observations carried out with the IRAM 30 m Telescope. IRAM is supported by INSU/CNRS (France), MPG (Germany) and IGN (Spain).
}

distribution similar to that of the optically thin dust emission are $\mathrm{N}_{2} \mathrm{H}^{+}$and $\mathrm{NH}_{3}$, consisting solely of nitrogen and hydrogen. This is in general attributed to the claim that their abundance is closely linked to that of molecular nitrogen (one of the main gas phase repositories of nitrogen) which is highly volatile and hence one of the last species to condense out (Bisschop et al. 2006). However $\mathrm{N}_{2}$ is only marginally more volatile than $\mathrm{CO}$ which is found observationally to condense out at densities of a few $10^{4} \mathrm{~cm}^{-3}$ (Tafalla et al. 2002, 2004) and it has thus been puzzling to have a scenario where C-containing species were completely frozen out but $\mathrm{N}_{2}$ not. This has led to a number of models aimed at retaining gas phase nitrogen for at least a short time after CO has disappeared, e.g. Flower et al. (2006), Akyilmaz et al. (2007), and Hily-Blant et al. (2010, hereafter Paper I). There have also been attempts to obtain convincing evidence for the existence in the gas phase of species containing both $\mathrm{C}$ and $\mathrm{N}$ at densities above a few $10^{5}$ to $10^{6} \mathrm{~cm}^{-3}$ at which CO depletes (e.g. Hily-Blant et al. 2008, and Paper I). A partial success in this regard was obtained by Hily-Blant et al. (2008) who found that the intensity of ${ }^{13} \mathrm{CN}(1-0)$ behaved in similar fashion to the dust emission in two of the densest cores: L183 and L1544. The implication of this is that at least some form of carbon, probably in the form of $\mathrm{CO}$, remains in the gas phase in these objects at densities above $10^{5} \mathrm{~cm}^{-3}$. Thus, a tentative conclusion is that the $\mathrm{CO}$ abundance at densities above $10^{5} \mathrm{~cm}^{-3}$ 
is much lower than the canonical value of $10^{-4}$ relative to $\mathrm{H}_{2}$ (Pontoppidan 2006), but nevertheless sufficiently high to supply carbon for minor species.

Putting this interpretation on a more solid foundation requires observation of possible tracers of the depleted region in cores carried out in a manner which will permit distinguishing species associated with high density gas from those present in the surrounding lower density envelope. This is complicated though perhaps facilitated by the gradients in molecular abundance due to depletion. While molecular abundances in general are expected to drop at high densities when depletion takes over, this is not necessarily true for all species at least within a limited density range (see the results for deuterated species discussed by Flower et al. 2006). However, proving that one is observing emission from high density gas involves either showing that one can detect transitions which require high densities to excite or showing that the emission comes from a compact region coincident with the dust emission peak. The former is rendered more difficult by the temperature gradients believed to exist in some cores (see e.g. Crapsi et al. 2005). The latter requires highly sensitive high angular resolution observations. A combination of the two is likely to be the best strategy.

One approach to these problems which has not been fully exploited to date is to make use of the hyperfine splitting present in essentially all low lying transitions of N-containing species. It is clear in the first place that the relative populations of hyperfine split levels of species such as $\mathrm{HCN}$ and $\mathrm{CN}$ are out of LTE in many situations (see Walmsley et al. 1982; Monteiro \& Stutzki 1986, and Paper I). Interpreting such anomalies requires accurate collisional rates between individual hyperfine split levels, but such rates can be determined from the rates between rotational levels (e.g. Monteiro \& Stutzki 1986) and clearly in principle, they allow limits to be placed on the density of the emitting region. It is also the case that species of relatively low abundance like $\mathrm{H}^{13} \mathrm{CN}$ are found to have rather minor deviations from LTE between hyperfine levels of the same rotational level (see Paper I) and in this case, one can determine the line excitation temperature and optical depth based on the relative hyperfine satellite intensities. While this is clearly doubtful procedure in that the non-LTE anomalies can cause errors in the inferred optical depth, it is nevertheless (as we shall discuss) an approach which can give a zero order estimate of relative rotational level populations and hence of the local density.

In the case of more abundant species such as the main isotopologues of HCN or CN, one finds that "self absorption" or absorption in foreground relatively low density material can obliterate any signal from the high density central regions of a core. However, from these tracers, one can in principle glean information on the kinematics of foreground layers of the core. Thus, one can hope to find evidence for infall. We indeed find in this study that profiles change in gradual fashion as a function of transition line strength and this does indeed yield evidence for either infall or expansion of foreground layers.

Here we extend the results of Paper I to three other cores which have been chosen on the basis of their CO depletion properties (see e.g. Bacmann et al. 2002; Brady Ford \& Shirley 2011). We in particular chose objects with large CO depletion holes indicative of a relatively large age. This might occur for example in sources where magnetic field is capable of slowing down or preventing collapse. We also used as a guide the angular size measured in the $\mathrm{N}_{2} \mathrm{H}^{+}(1-0)$ transition by Caselli et al. (2002b) which is thought to correspond roughly to the area of CO depletion. Thus, we chose the cores L1498 and TMC 2 which appear to have large $\mathrm{CO}$ depletion holes $(0.08$ parsec in the case of TMC 2). As a comparison source, we included in our study L1521E, which shows little or no depletion in C-bearing species (Tafalla \& Santiago 2004).

In this paper then, we present IRAM $30 \mathrm{~m}$ observations of the $J=1 \rightarrow 0$ transition of $\mathrm{HCN}, \mathrm{H}^{13} \mathrm{CN}$ and $\mathrm{HN}^{13} \mathrm{C}$ along the major and the minor axes of the three selected sources, L1498, L1521E and TMC 2, as well as the $J=1 \rightarrow 0$ transition of $\mathrm{N}_{2} \mathrm{H}^{+}$and the $J=2 \rightarrow 1$ transition of $\mathrm{C}^{18} \mathrm{O}$ towards TMC 2. In Sect. 2, we discuss the observational and data reduction procedures, summarising the results in Sect. 3. In Sect. 4 we compare the distribution of the line emission with respect to the dust emission, investigating about the presence of depletion. In Sect. 5 we describe the evidence of the asymmetry of the HCN(1-0) line profiles. In Sect. 6 we explain the methods used for determining the excitation temperature, while in Sect. 7 we give the estimated column densities and abundances for the observed lines. In Sect. 8 we give our conclusions. Comment on non-LTE hyperfine populations are provided in Appendix A, while in Appendix B we summarise the spectroscopic data and observational parameters of the observed molecules.

\section{Observations}

L1498, L1521E, and TMC 2 were observed in July 2008, using frequency switching and raster mode, with a spacing of $20^{\prime \prime}$, $25^{\prime \prime}$ and $50^{\prime \prime}$, respectively, along the major and the minor axes. The axes were identified from the continuum emission maps showed in Tafalla et al. (2002) for L1498, Tafalla et al. (2004) for L1521E, and Crapsi et al. (2005) for TMC 2. Observing parameters are given in Table B.3.

Observations of the $89 \mathrm{GHz} \mathrm{HCN}(1-0)$ and the $86 \mathrm{GHz}$ $\mathrm{H}^{13} \mathrm{CN}(1-0)$ multiplets (see Table B.1) plus the $87 \mathrm{GHz}$ $\mathrm{HN}^{13} \mathrm{C}(1-0)$ multiplet (see Table B.2) were carried out using the VESPA autocorrelator with $20 \mathrm{kHz}$ channel spacing (corresponding to about $0.069 \mathrm{~km} \mathrm{~s}^{-1}$ ) with $40 \mathrm{MHz}$ bandwidth for $\mathrm{HCN}(1-0)$ and $20 \mathrm{MHz}$ bandwidth for $\mathrm{H}^{13} \mathrm{CN}(1-0)$ and $\mathrm{HN}^{13} \mathrm{C}(1-0)$. The final rms, in $T_{\mathrm{mb}}$ unit is $\sigma_{\mathrm{T}} \sim 50 \mathrm{mK}$, $\sim 20 \mathrm{mK}$, and $\sim 30 \mathrm{mK}$ for $\mathrm{HCN}(1-0), \mathrm{H}^{13} \mathrm{CN}(1-0)$, and $\mathrm{HN}^{13} \mathrm{C}(1-0)$, respectively.

Observations of the $93 \mathrm{GHz} \mathrm{N} \mathrm{H}^{+}(1-0)$ multiplet and of the $219 \mathrm{GHz} \mathrm{C} \mathrm{C}^{18} \mathrm{O}(2-1)$ line were carried out in TMC 2 using $10 \mathrm{kHz}$ channel spacing with $40 \mathrm{MHz}$ bandwidth for $\mathrm{N}_{2} \mathrm{H}^{+}(1-0)$ and $20 \mathrm{kHz}$ channel spacing with $40 \mathrm{MHz}$ bandwidth for $\mathrm{C}^{18} \mathrm{O}(2-1)$. For $\mathrm{N}_{2} \mathrm{H}^{+}$the final rms, in $T_{\mathrm{mb}}$ units, in channels of width $\delta v=0.031 \mathrm{~km} \mathrm{~s}^{-1}$, is $\sigma_{\mathrm{T}} \sim 100 \mathrm{mK}$ while for $\mathrm{C}^{18} \mathrm{O}(2-1)$, the final rms (channels of width $\delta v=0.027 \mathrm{~km} \mathrm{~s}^{-1}$ ) is $\sigma_{\mathrm{T}} \sim 250 \mathrm{mK}$.

Data reduction and analysis were completed using the CLASS software of the GILDAS ${ }^{1}$ facility, developed at the IRAM and the Observatoire de Grenoble. In what follows, all temperatures are on the main-beam scale, $T_{\mathrm{mb}}=F_{\mathrm{eff}} T_{\mathrm{A}}^{*} / B_{\mathrm{eff}}$, where $T_{\mathrm{A}}^{*}$ is the antenna temperature corrected for atmospheric absorption, while $B_{\text {eff }}$ and $F_{\text {eff }}$ are the beam and the forward efficiencies, respectively (see Table B.3 for the numerical values of efficiencies).

\section{Observational results}

Figure 1 shows the continuum emission of the three cores together with the positions mapped in lines described in Sect. 2. The selected sources have already been widely observed in the past as possible tracers of conditions in the high density core

${ }^{1}$ http://www.iram.fr/IRAMFR/GILDAS 
L1498

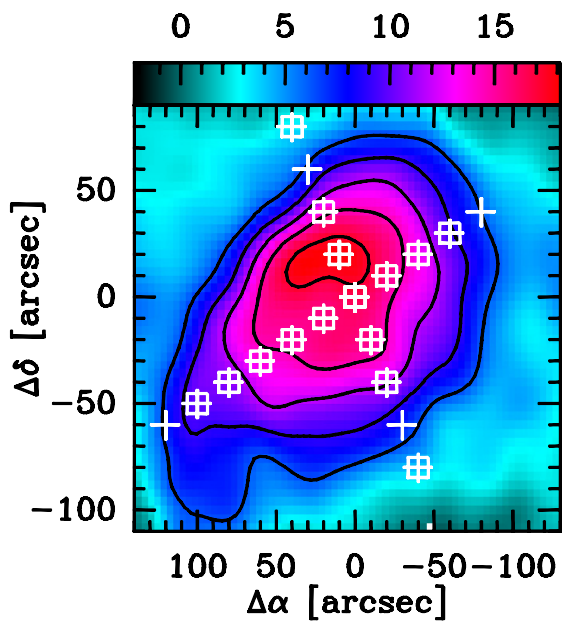

L1521E
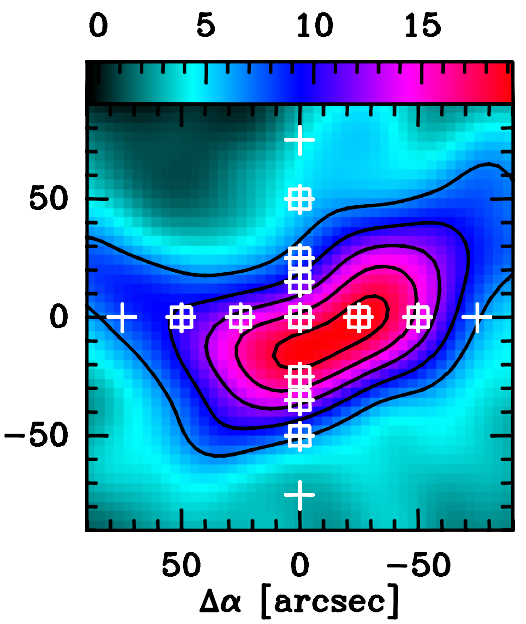

TMC 2
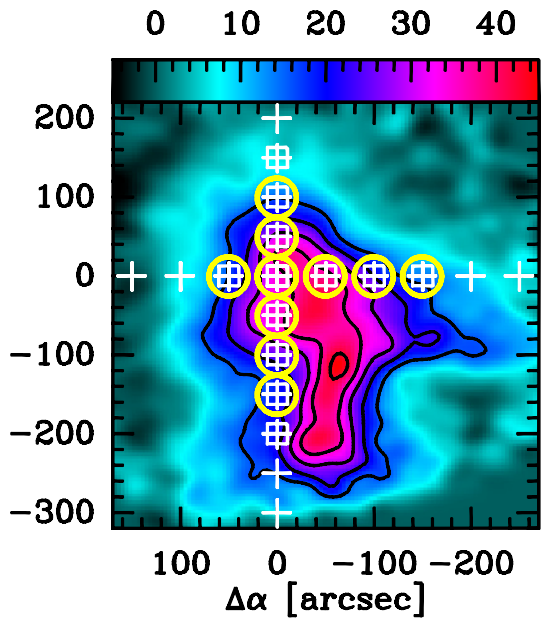

Fig. 1. Observed positions in L1498 (left panel), L1521E (middle panel), and TMC 2 (right panel), superposed on the dust emission map smoothed to $28^{\prime \prime}$, from Tafalla et al. $(2002,2004)$, and Crapsi et al. (2005), respectively. Mapped positions in $\mathrm{HCN}(1-0)($ white $\mathrm{crosses})$, $\mathrm{H}^{13} \mathrm{CN}(1-0)$ and $\mathrm{HN}^{13} \mathrm{C}(1-0)$ (white squares), and $\mathrm{N}_{2} \mathrm{H}^{+}(1-0)$ (yellow circles). Contours represent 35, 50, 65, 80 and 95 per cent of the peak value of the dust emission which is 18.0, 21.0, and $45.0 \mathrm{mJy} /\left(11^{\prime \prime}\right.$ beam $)$ for L1498, L1521E, and TMC 2, respectively. The $(0,0)$ position corresponds to $\alpha(2000)=$ $04^{\mathrm{h}} 10^{\mathrm{m}} 51.5^{\mathrm{s}}, \delta(2000)=25^{\circ} 09^{\prime} 58^{\prime \prime}$ for L1498, to $\alpha(2000)=04^{\mathrm{h}} 29^{\mathrm{m}} 15.7^{\mathrm{s}}, \delta(2000)=26^{\circ} 14^{\prime} 05^{\prime \prime}$ for L1521E, and to $\alpha(2000)=04^{\mathrm{h}} 32^{\mathrm{m}} 48.7^{\mathrm{s}}$, $\delta(2000)=24^{\circ} 25^{\prime} 12^{\prime \prime}$ for TMC 2 .

nucleus. We succeeded in observing $\mathrm{HN}^{13} \mathrm{C}(1-0)$ and the three hyperfine components of $\mathrm{HCN}(1-0)$ and $\mathrm{H}^{13} \mathrm{CN}(1-0)$.

Figure 2 shows a comparison of the line profiles for the different tracers. We plotted the weakest $(F=0 \rightarrow 1)$ component of $\mathrm{HCN}(1-0)$ at $88633.936 \mathrm{MHz}$, the strongest $(F=2 \rightarrow 1)$ component of $\mathrm{H}^{13} \mathrm{CN}(1-0)$ at $86340.184 \mathrm{MHz}, \mathrm{HN}^{13} \mathrm{C}(1-0)$ at $87090.675 \mathrm{MHz}$, the isolated component $\left(F_{1}, F=0,1 \rightarrow 1,2\right)$ of $\mathrm{N}_{2} \mathrm{H}^{+}(1-0)$ at $93176.2650 \mathrm{MHz}$, and the $\mathrm{C}^{18} \mathrm{O}(2-1)$ transition at $219560.319 \mathrm{MHz}$. We used the components enumerated here also for the comparison between line and dust emission described below, supposing them to be the most optically thin. The offsets for each source are relative to the dust peak emission (see caption of Fig. 1).

There are clear trends in the line widths which we derive for our three sources with values of order $0.2 \mathrm{~km} \mathrm{~s}^{-1}$ for L1498, $0.3 \mathrm{~km} \mathrm{~s}^{-1}$ for L1521E, and $0.45 \mathrm{~km} \mathrm{~s}^{-1}$ for TMC 2. Besides, we noticed that there is a reasonable agreement between the systemic velocity, $V_{\mathrm{LSR}}$, of $\mathrm{N}_{2} \mathrm{H}^{+}$and $\mathrm{H}^{13} \mathrm{CN}$ to within $0.08 \mathrm{~km} \mathrm{~s}^{-1}$, while line widths of $\mathrm{H}^{13} \mathrm{CN}$ seem larger by $0.05 \mathrm{~km} \mathrm{~s}^{-1}$ with respect to $\mathrm{N}_{2} \mathrm{H}^{+}$, but this may be due to ${ }^{13} \mathrm{C}$ hyperfine splitting (Schmid-Burgk et al. 2004).

There is a clear difference between the $\mathrm{HCN}$ and $\mathrm{H}^{13} \mathrm{CN}$ profiles in the three sources: while in L1521E, the HCN profile is broader than its isotopologue, in L1498 and TMC 2, HCN and $\mathrm{H}^{13} \mathrm{CN}$ have very different profiles, presumably because of high optical depth in HCN.

All these molecules show hyperfine structure, although in the case of $\mathrm{HN}^{13} \mathrm{C}$ it is not possible to avoid the blending of the components, and the hyperfine fitting has been conducted using the HFS method in CLASS. To fit this line, we followed van der Tak et al. (2009) who found that it consists of eleven hyperfine components which can however be reduced to four "effective" components. As an instance, in the upper panel of Fig. 3, we show the fit of the $\mathrm{HN}^{13} \mathrm{C}$ line at the offset $(10,20)$ towards L1498 together with the four distinguishable hyperfine components. The fit gives a value for the total optical depth equal to $\tau=4.58 \pm 0.32$ and all the observed points show similar values, as shown in Fig. 4, with an average of $\langle\tau\rangle=5.16 \pm 0.86$. Also for $\mathrm{H}^{13} \mathrm{CN}$ we found a good simultaneous fit of the three
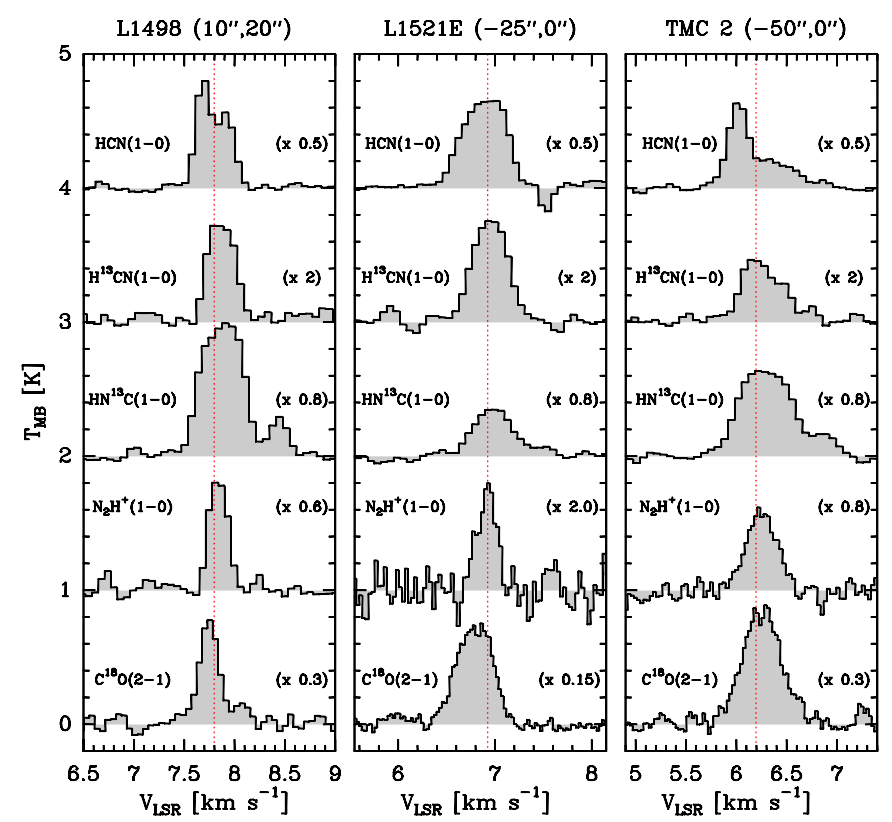

Fig. 2. Emission line for the different tracers of the source sample towards the dust emission peak. The $88633 \mathrm{MHz}$ component of $\mathrm{HCN}(1-0)$ is shown together with the $86340 \mathrm{MHz}$ component of $\mathrm{H}^{13} \mathrm{CN}(1-0), \mathrm{HN}^{13} \mathrm{C}(1-0)$, the $93176 \mathrm{MHz}$ isolated component of $\mathrm{N}_{2} \mathrm{H}^{+}(1-0)$, and $\mathrm{C}^{18} \mathrm{O}(2-1)$. Spectra have been multiplied by a scaling factor to allow the simultaneous comparison. Red dotted lines show the systemic LSR velocity of the sources evaluated from the welldetermined frequencies of $\mathrm{N}_{2} \mathrm{H}^{+}(1-0)$, see Pagani et al. (2009).

hyperfine components (see lower panel of Fig. 3) and, as $\mathrm{HN}^{13} \mathrm{C}$, this line is somewhat optically thick with a total optical depth of $\tau=3.11 \pm 0.77$ at the offset $(10,20)$ and a mean optical depth of $\langle\tau\rangle=4.34 \pm 1.15$. Hence one can expect the optical depth in $\mathrm{HCN}$ hyperfine components to be of order 30-100 assuming the canonical isotopic ratio for $\left[{ }^{12} \mathrm{C}\right] /\left[{ }^{13} \mathrm{C}\right]$ of 68 (Milam et al. 2005). 

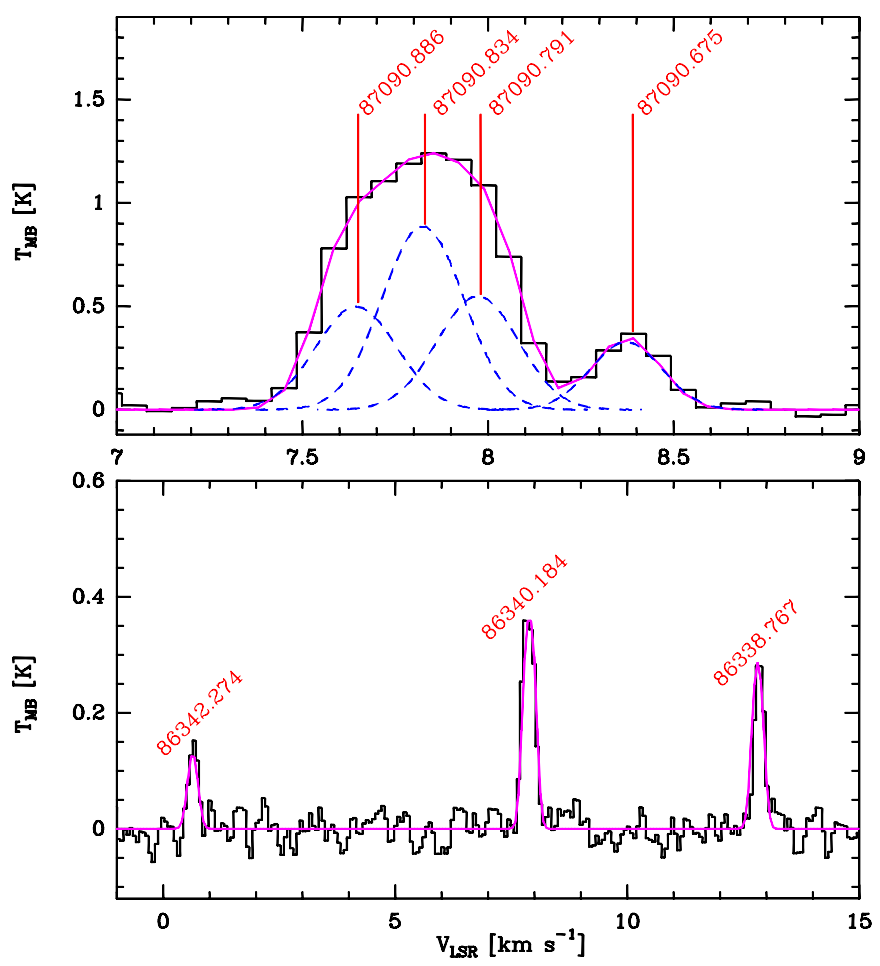

Fig. 3. $\mathrm{HN}^{13} \mathrm{C}(1-0)$ (upper panel) and $\mathrm{H}^{13} \mathrm{CN}(1-0)$ (lower panel) emission towards L1498 at the offset $(10,20)$; black solid histogram, hyperfine components used for the fit, blue dashed lines, centred at the frequencies listed in Table B.2, and line fit, magenta solid line.

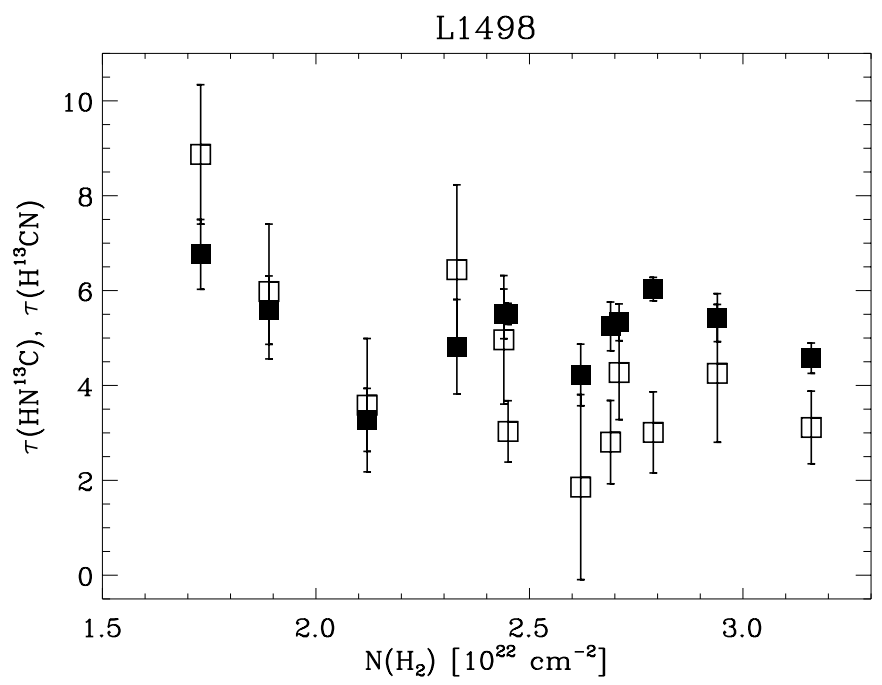

Fig. 4. Total optical depth of $\mathrm{HN}^{13} \mathrm{C}(1-0)$, solid squares, and $\mathrm{H}^{13} \mathrm{CN}(1-0)$, empty squares, for all the observed positions in L1498 as a function of the molecular hydrogen column density.

\section{Probing the presence of depletion}

In this section, we study the dependence of the integrated intensity, $W$, of the $\mathrm{HCN}, \mathrm{H}^{13} \mathrm{CN}$ and $\mathrm{HN}^{13} \mathrm{C}$ transitions on offset from the dust emission peaks. In the case of $\mathrm{HCN}$, we consider the weakest $F=0 \rightarrow 1$ line and in the case of $\mathrm{H}^{13} \mathrm{CN}$, the strongest $F=2 \rightarrow 1$ satellite. The observational results are shown in Figs. 6-8 where we compare the observed intensity of these lines with the dust emission which is taken to be representative of the $\mathrm{H}_{2}$ column density. It is useful first to comment

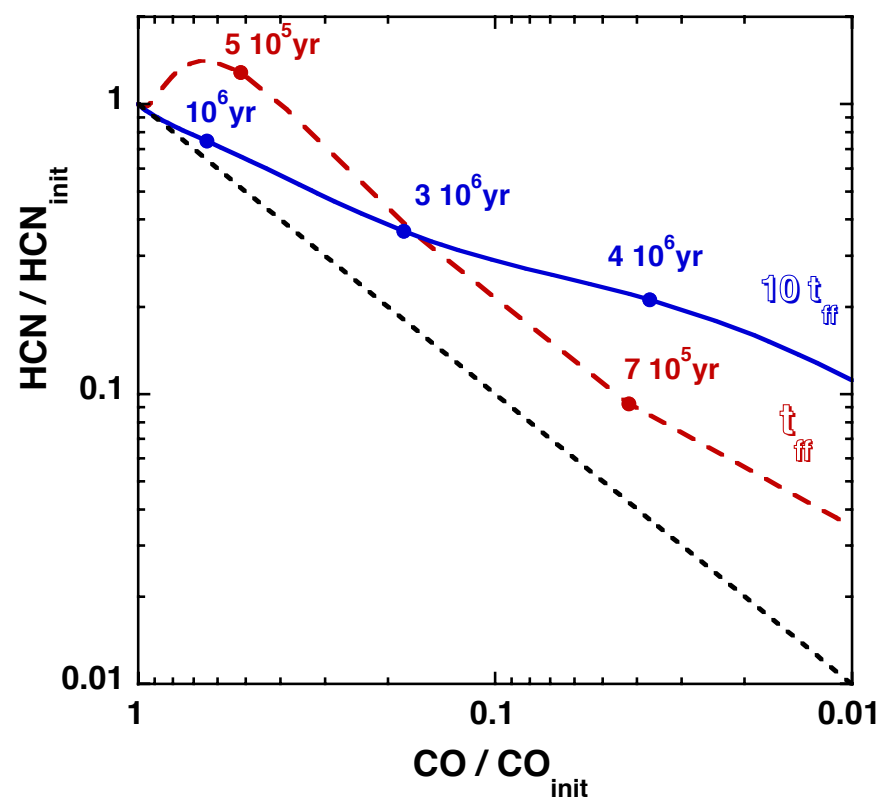

Fig. 5. The HCN depletion as a function of the CO depletion during the collapse, computed by the gravitational collapse model of Paper I, see their Fig. 8. Values are normalised with respect to the initial abundances. Each point is labelled with the time of the collapsing gas. Two models are shown: a free fall model labelled $t_{\mathrm{ff}}$ (red dashed curve) and a collapsing model with a time scale multiplied by a factor ten, labelled $10 t_{\mathrm{ff}}$ (blue solid curve). The black dotted curve shows the positions of equal depletion for $\mathrm{CO}$ and $\mathrm{HCN}$.

briefly on what one can expect qualitatively to learn from such studies.

One notes in the first place that optically thick transitions, due to scattering and line saturation, are naturally likely to have a broader spatial distribution than thin (or very moderate optical depth) transitions. Thus, the $\operatorname{HCN}(F=0 \rightarrow 1)$ transition can be expected to be roughly an order of magnitude more optically thick than $\mathrm{H}^{13} \mathrm{CN}(F=2 \rightarrow 1)$ assuming the local interstellar $\left[{ }^{12} \mathrm{C}\right] /\left[{ }^{13} \mathrm{C}\right]$ ratio (Milam et al. 2005) and no fractionation. One thus expects a broader spatial distribution of $\mathrm{HCN}$ than $\mathrm{H}^{13} \mathrm{CN}$ and indeed this is confirmed by the results shown in all the three sources. For example, the half power size of HCN in L1498 along the NW-SE cut is $170^{\prime \prime}$ as compared with $120^{\prime \prime}$ for $\mathrm{H}^{13} \mathrm{CN}$ and $\mathrm{HN}^{13} \mathrm{C}$, and $180^{\prime \prime}$ for the dust emission. One concludes that the ${ }^{13} \mathrm{C}$ substituted isotopologue is a much better tracer of $\mathrm{HCN}$ column density than the more abundant form even when using the weakest hyperfine component.

However, one also notes from these figures that the $\mathrm{C}^{18} \mathrm{O}$ distribution is essentially flat as has been seen in a variety of studies (Tafalla et al. 2002, 2004). This has been attributed to depletion of $\mathrm{CO}$ at densities $n\left(\mathrm{H}_{2}\right)$ above a critical value of order a few times $10^{4} \mathrm{~cm}^{-3}$ and our observations are entirely consistent with this. It is also true however (see also Tafalla et al. 2006, and Paper I) that species like $\mathrm{H}^{13} \mathrm{CN}$ and $\mathrm{HN}^{13} \mathrm{C}$, while they clearly have different spatial distributions from the dust emission, have half power sizes which are roughly similar (see results for L1498 above). Such effects clearly do not apply to $\mathrm{N}_{2} \mathrm{H}^{+}$which does not contain carbon and which in general has been found to have a spatial distribution similar to that of the dust (Tafalla et al. 2006). However, one sees in Figs. $6-8$ that $\mathrm{HN}^{13} \mathrm{C}$ and $\mathrm{H}^{13} \mathrm{CN}$ have a spatial distribution closer to that of the dust emission and we conclude that these two isotopologues may often be very useful tracers of kinematics as $\mathrm{N}_{2} \mathrm{H}^{+}$at densities above the critical 


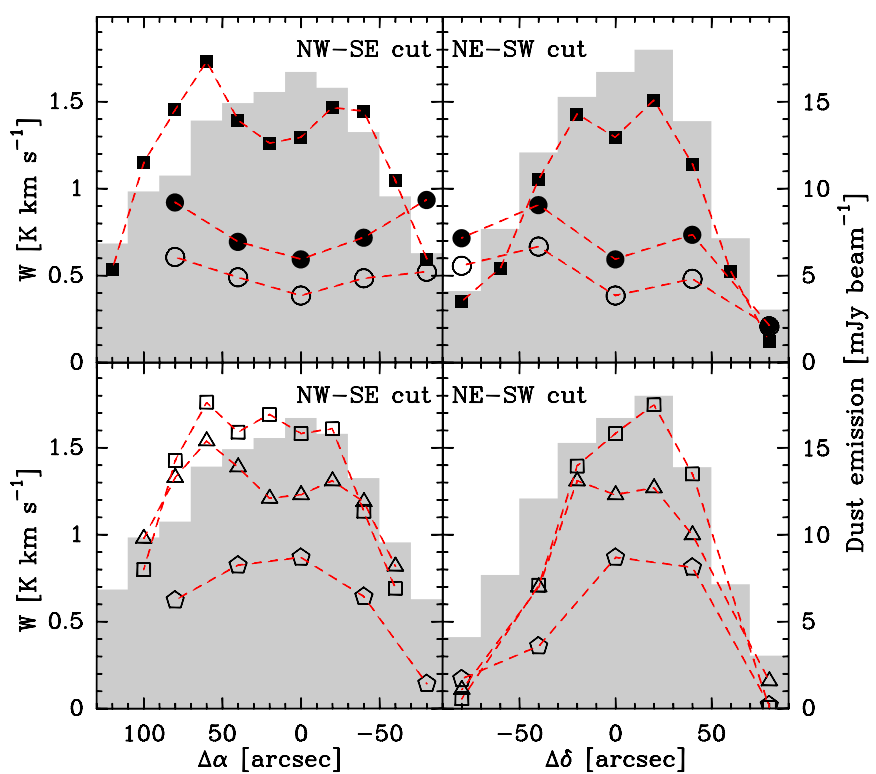

Fig. 6. Comparison between the dust emission (gray histograms) and the integrated intensity of the observed species along the two cuts in L1498. Upper panels: solid squares, $\mathrm{HCN}(1-0)[\times 3]$; empty circles, $\mathrm{C}^{18} \mathrm{O}(1-0)[\times 0.8]$; solid circles, $\mathrm{C}^{18} \mathrm{O}(2-1)[\times 1.4]$. Lower panels: empty triangles, $\mathrm{H}^{13} \mathrm{CN}(1-0)[\times 10]$; empty squares, $\mathrm{HN}^{13} \mathrm{C}(1-0)$ [×2.5]; empty pentagons, $\mathrm{N}_{2} \mathrm{H}^{+}(1-0)[\times 3]$. The typical error on the integrated intensity is about $20 \mathrm{mK} \mathrm{km} \mathrm{s}$.

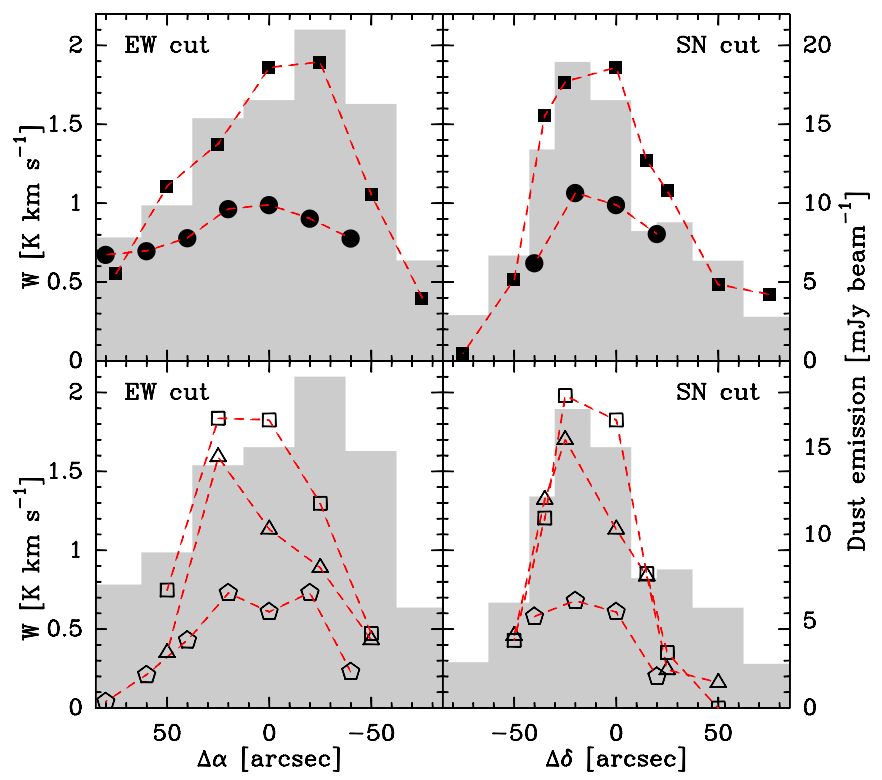

Fig. 7. Comparison between the dust emission (gray histograms) and the integrated intensity of the observed species along the two cuts in L1521E. Upper panels: solid squares, $\operatorname{HCN}(1-0)[\times 2.5]$; solid circles, $\mathrm{C}^{18} \mathrm{O}(2-1)[\times 0.4]$. Lower panels: empty triangles, $\mathrm{H}^{13} \mathrm{CN}(1-0)[\times 27]$; empty squares $\mathrm{HN}^{13} \mathrm{C}(1-0)$ [ $\times 5.5$ ]; empty pentagons, $\mathrm{N}_{2} \mathrm{H}^{+}(1-0)[\times 10]$. The typical error on the integrated intensity is about $20 \mathrm{mK} \mathrm{km} \mathrm{s}^{-1}$.

values at which $\mathrm{CO}$ depletes. It is possible that $\mathrm{CO}$, while depleted by roughly an order of magnitude relative to the canonical $[\mathrm{CO}] /\left[\mathrm{H}_{2}\right]$ abundance ratio of $10^{-4}$, is nevertheless sufficiently abundant to account for minor species such as HCN and HNC.

Finally, we note from Fig. 8 that towards TMC 2, where the dust peak $\mathrm{H}_{2}$ column density is larger than in the other two sources, there seems to be a good general accord between the

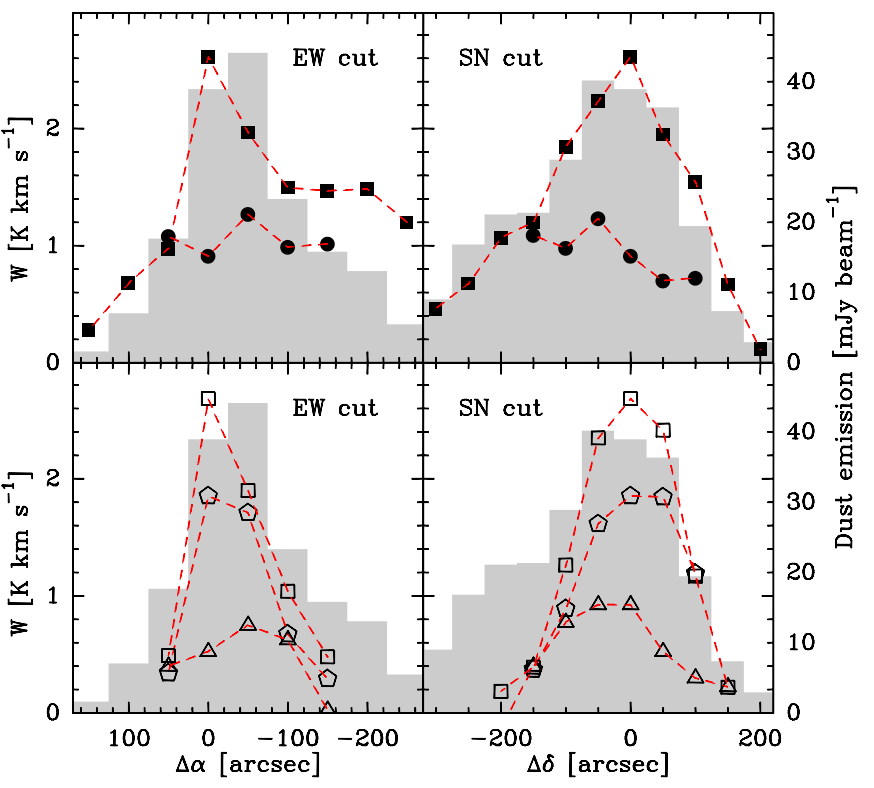

Fig. 8. Comparison between the dust emission (gray histograms) and the integrated intensity of the observed species along the two cuts in TMC 2. Upper panels: solid squares, $\operatorname{HCN}(1-0)[\times 4]$; solid circles, $\mathrm{C}^{18} \mathrm{O}(2-1)$. Lower panels: empty triangles, $\mathrm{H}^{13} \mathrm{CN}(1-0)[\times 25]$; empty squares, $\mathrm{HN}^{13} \mathrm{C}(1-0)[\times 3.5]$; empty pentagons, $\mathrm{N}_{2} \mathrm{H}^{+}(1-0)[\times 4.5]$. The typical error on the integrated intensity is about $20 \mathrm{mK} \mathrm{km} \mathrm{s}^{-1}$.

dust emission and the intensity of $\mathrm{HN}^{13} \mathrm{C}, \mathrm{H}^{13} \mathrm{CN}$, and $\mathrm{N}_{2} \mathrm{H}^{+}$. The $\mathrm{CO}$ however has again a flat distribution suggesting once more that it is depleted in the vicinity of the dust peak. There is no reason in this source to suppose that $\mathrm{N}_{2} \mathrm{H}^{+}$traces the high density gas around the core nucleus better than the ${ }^{13} \mathrm{C}$ substituted isotopologues of $\mathrm{HCN}$ and HNC. There are however slight differences close to the dust peak between the different species which are perhaps attributable to excitation and optical depth effects, but could also be caused by the depletion which presumably all molecules undergo if the density is sufficiently high. We conclude that cyanides and isocyanides are useful tracers of gas with densities around $10^{5} \mathrm{~cm}^{-3}$.

We note that models of a collapsing prestellar core indeed suggest that $\mathrm{HCN}$ and $\mathrm{HNC}$ should remain with appreciable gas-phase abundance at an epoch when the $\mathrm{CO}$ abundance has depleted to a few percent of the canonical value of $10^{-4}$ relative to $\mathrm{H}_{2}$. To illustrate the differential freeze out of $\mathrm{HCN}$ relative to that of $\mathrm{CO}$ found in the chemical models, Fig. 5 displays the depletion of $\mathrm{HCN}$ as a function of the depletion of $\mathrm{CO}$ computed in the collapsing gas, starting from steady-state abundances at a density of $10^{4} \mathrm{~cm}^{-3}$. The numerical gravitational collapse model is described in Sect. 6.2 of Paper I. Two models are shown for two different collapse time scale: free-fall time $\left(t_{\mathrm{ff}}\right)$ and $10 t_{\mathrm{ff}}$. It may be seen from Fig. 5 that the depletion of $\mathrm{HCN}$ relative to that of $\mathrm{CO}$ strongly depends on the collapse time scale: the model shows that $\mathrm{HCN}$ is depleted on longer timescales than $\mathrm{CO}$, in particular if the dynamical timescale is several free fall times. HNC, not shown in the plot, has the same behaviour of $\mathrm{HCN}$.

\section{Line profiles}

In this section, we discuss the behaviour of the HCN line profiles as a function of optical depth. It is clear from Fig. 2 that towards L1498 and TMC 2, the $F=0 \rightarrow 1$ component of HCN has a profile skewed to the blue relative to $F=2 \rightarrow 1$ of $\mathrm{H}^{13} \mathrm{CN}$ and 

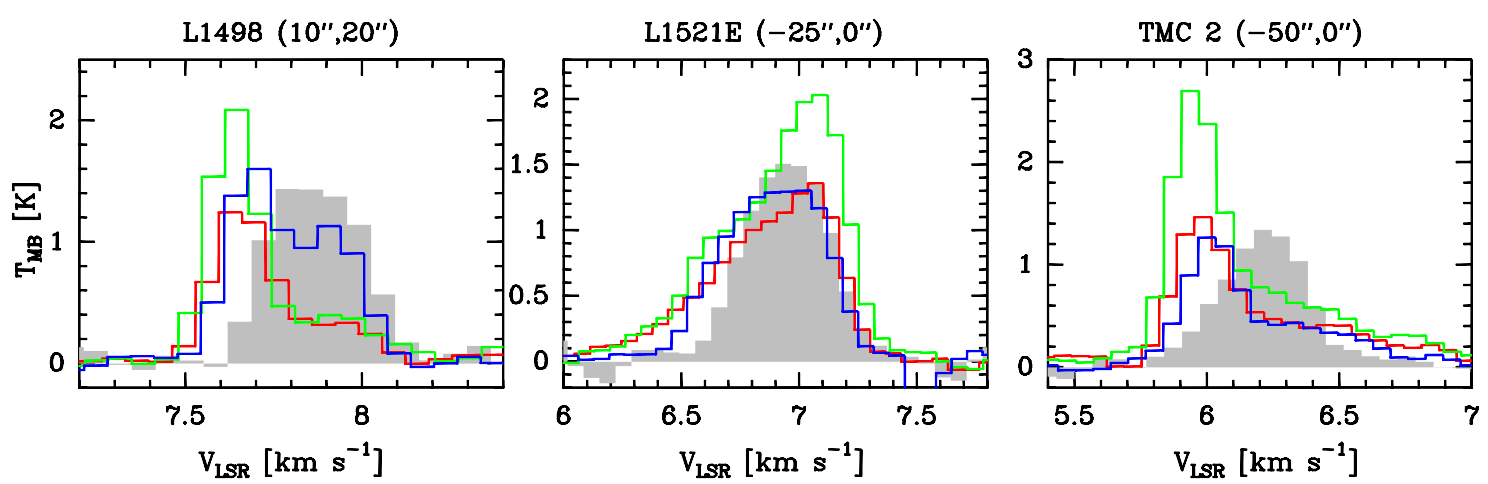

Fig. 9. Superposition of the three hyperfine components of $\operatorname{HCN}(1-0)(F=1 \rightarrow 1$, red line; $F=2 \rightarrow 1$, green line; $F=0 \rightarrow 1$, blue line) with the strongest component $\left(F=2 \rightarrow 1\right.$, gray histograms) of $\mathrm{H}^{13} \mathrm{CN}(1-0)$ [ $\times 4$ ], see Table B.1 for component indices. In order to compare line shapes and intensities, components have been shifted in frequency.
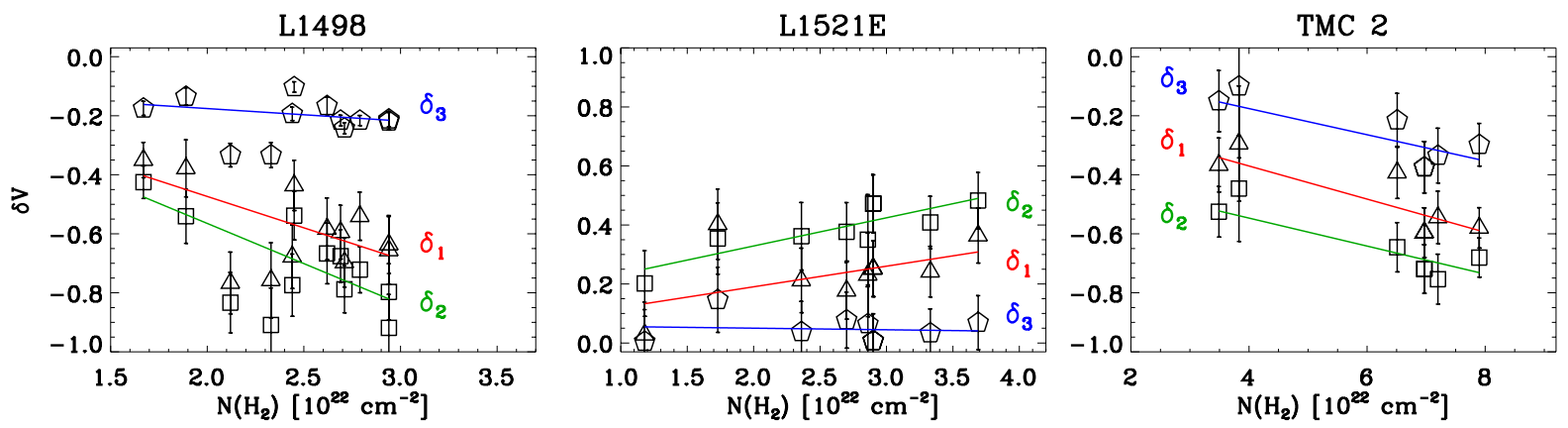

Fig. 10. Degree of skewness as a function of the column density of molecular hydrogen: $\delta_{i}=\left(V_{i}-V_{\mathrm{H}^{13} \mathrm{CN}}\right) / \Delta V_{\mathrm{H}^{13} \mathrm{CN}}$, where $i=1,2,3$ is the $i$ th component of $\mathrm{HCN}(1-0)$, and $V_{\mathrm{H}^{13} \mathrm{CN}}$ and $\Delta V_{\mathrm{H}^{13} \mathrm{CN}}$ are the velocity and the line width of component $2(F=2 \rightarrow 1$, the strongest) of the isotopologue $\mathrm{H}^{13} \mathrm{CN}(1-0)$. Values of $\delta V$ from observations of component $1(F=1 \rightarrow 1)$, triangles; component $2(F=2 \rightarrow 1)$, squares; component $3(F=0 \rightarrow 1)$, pentagons. See also Table B.1 for component indices.

it is tempting to interpret this as being due to absorption in a foreground infalling layer. Figure 9 illustrates this well showing all three $\mathrm{HCN}$ components compared with the strongest $F=$ $2 \rightarrow 1$ component of $\mathrm{H}^{13} \mathrm{CN}$. One sees that there is in fact a progression going from the highly skewed optically thick $F=$ $2 \rightarrow 1$ component of $\mathrm{HCN}$ to the essentially symmetric profile of $\mathrm{H}^{13} \mathrm{CN}$. In the following, we attempt to quantify this trend.

Looking at Fig. 9, related to the three hyperfine components of $\mathrm{HCN}$ and the $\mathrm{H}^{13} \mathrm{CN}(F=2 \rightarrow 1)$ component, one can qualitatively argue that the greater the relative intensity of a line, the higher is the skewness degree. It can be seen that the $\mathrm{H}^{13} \mathrm{CN}(F=2 \rightarrow 1)$ component (gray histogram) is fairly symmetric and indeed, as seen earlier, its optical depth is not high since it is next to optically thin limit, but HCN components are skewed towards the blue in L1498 and TMC 2, while in L1521E they are skewed towards the red.

The superposition of the different hyperfine components of $\mathrm{HCN}$ led us to the discovery of a correlation between the line profile and its intensity. In particular, a red-absorbed line profile (that is skewed towards the blue) is a hint for the presence of an outer layer which is absorbing the emission of the inner layer while moving away from the observer, suggesting infall motions. Conversely, a blue-absorbed line profile (that is skewed towards the red) is an indication of the motion of the outer absorbing layer towards the observer, that is outflow motions.

To quantify these deviations from the expected Gaussian shape in the optically thin limit, we compared the asymmetry degree towards the different positions mapped, using the definition of skewness, $\delta V$, given by Mardones et al. (1997)

$\delta V=\frac{V_{\text {thick }}-V_{\text {thin }}}{\Delta V_{\text {thin }}}$,

where $V_{\text {thick }}$ and $V_{\text {thin }}$ are the velocities at the peak of the optically thick and thin components, respectively, while $\Delta V_{\text {thin }}$ is the line width of the thin component. The normalisation of the velocity difference with $\Delta V_{\text {thin }}$ reduces bias arising from lines of different width measured in different sources, allowing a more realistic comparison of the values of $\delta V$ in our sample. Velocities and line widths have been determined by Gaussian fits; in the event that optically-thick line profiles were double peaked, we fitted these two components with two Gaussians, assigning to $V_{\text {thick }}$ the central velocity of the Gaussian relative to the stronger of the two peaks. We supposed the $F=2 \rightarrow 1$ component of $\mathrm{H}^{13} \mathrm{CN}$ to be optically thin, so that $V_{\text {thin }}=V_{\mathrm{H}^{13} \mathrm{CN}}$ and $\Delta V_{\text {thin }}=\Delta V_{\mathrm{H}^{13} \mathrm{CN}}$. A value of $\delta V$ lower than zero suggests a red absorption of the line, while a positive value a blue absorption.

Figure 10 shows the values of the skewness degree, $\delta V$, for the three hyperfine components of $\mathrm{HCN}$ as a function of the column density of molecular hydrogen for our source sample. Hence we confirm the suggestion made earlier that: (i) the absolute value of $\delta V$ is greater for the strongest hyperfine components, see also Fig. 11, in fact, in all the three sources, $\mid \delta V(F=$ $2 \rightarrow 1)|>| \delta V(F=1 \rightarrow 1)|>| \delta V(F=0 \rightarrow 1) \mid$; (ii) in L1498 and TMC 2, the emission lines are red-absorbed, since $\delta V<0$, and this is a hint for the presence of infall motions; 

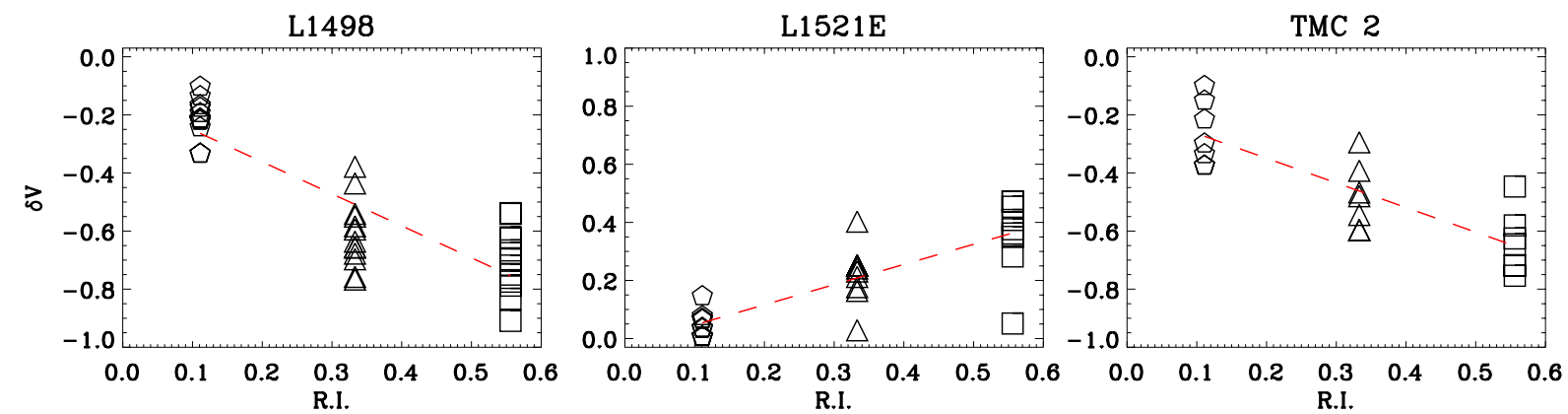

Fig. 11. Degree of skewness as a function of the relative intensities of the hyperfine components of HCN(1-0). Linear regressions emphasise the relationship between $\delta V$ and line strength (dashed red lines). Values of $\delta V$ from observations of the $F=1 \rightarrow 1$ component (triangles), the $F=2 \rightarrow 1$ component (squares), and the $F=0 \rightarrow 1$ component (pentagons).

(iii) in L1521E, the emission lines are blue-absorbed $(\delta V>0)$, suggesting expansion; (iv) as expected, $\delta V$ decreases from the centre to the outer part of the cores, that is for decreasing values of $N\left(\mathrm{H}_{2}\right)$, dropping with line intensities, though sometimes not much; (v) $\delta V(F=0 \rightarrow 1)$ for $\mathrm{HCN}$ seems to be rather independent of the $\mathrm{H}_{2}$ column density, being almost constant for L1498 and L1521E, probably because this hyperfine component is the weakest line and it is closer to the optically thin limit.

\section{Excitation temperature results}

Even though real deviations from LTE populations are present (see Appendix A), the LTE assumption is an approximation which is useful for many purposes and we calculated the excitation temperature, $T_{\mathrm{ex}}$, from a simultaneous LTE fit of the hyperfine components in the observed species, using the measured intensity of optically thick transitions. The main-beam temperature is given by

$T_{\mathrm{mb}}=f_{\mathrm{B}}\left[J_{v}\left(T_{\mathrm{ex}}\right)-J_{v}\left(T_{\mathrm{bb}}\right)\right]\left(1-\mathrm{e}^{-\tau}\right)$,

where $J_{v}(T)=T_{0} /\left[\exp \left(T_{0} / T\right)-1\right]$ is the Planck-corrected brightness temperature and $T_{\mathrm{bb}}=2.73 \mathrm{~K}$ is the temperature of the cosmic background. $T_{0} \equiv h v / k$, where $v$ is the transition frequency, and $h$ and $k$ represent Planck's and Boltzmann's constants, respectively. If both the source and the beam are Gaussian shaped, the beam filling factor, $f_{\mathrm{B}}$, is given by

$f_{\mathrm{B}}=\frac{\Omega_{\mathrm{S}}}{\Omega_{\mathrm{B}}+\Omega_{\mathrm{S}}}$,

where $\Omega_{\mathrm{B}}=1.133 \theta_{\mathrm{B}}^{2}$ and $\Omega_{\mathrm{S}}=1.133 \theta_{\mathrm{S}}^{2}$ denote the solid angles covered by the beam and the source, respectively, while $\theta_{\mathrm{B}}$ and $\theta_{\mathrm{S}}$ are the half-power beamwidth of the beam and the source, respectively, this latter evaluated by taking the line intensities stronger than the $50 \%$ of the peak value. We found $f_{\mathrm{B}}$ to be equal to unity for most of the tracers, because $\Omega_{\mathrm{S}}$ is at least one order of magnitude greater than $\Omega_{\mathrm{B}}$, except for $\mathrm{H}^{13} \mathrm{CN}$ and $\mathrm{HN}^{13} \mathrm{C}$ in $\operatorname{L} 1521 \mathrm{E}\left(f_{\mathrm{B}}=0.88\right)$.

The correct determination of the excitation temperature for $\mathrm{HN}^{13} \mathrm{C}$ and $\mathrm{H}^{13} \mathrm{CN}$ is fundamental. In fact, the gas density of the source sample $\left(\sim 10^{5} \mathrm{~cm}^{-3}\right.$, see Tafalla et al. 2004; Tafalla \& Santiago 2004; and Crapsi et al. 2005, for L1498, L1521E, and TMC 2, respectively) corresponds to a region where the $T_{\mathrm{ex}}$ of the two isotopologues changes rapidly with density, namely between the radiation- and collision-dominated density ranges. We can thus in principle use our $T_{\text {ex }}$ determinations to constrain the density and temperature in the region where the $\mathrm{H}^{13} \mathrm{CN}$ and $\mathrm{HN}^{13} \mathrm{C}$ lines are formed. Since these transitions are emitted in

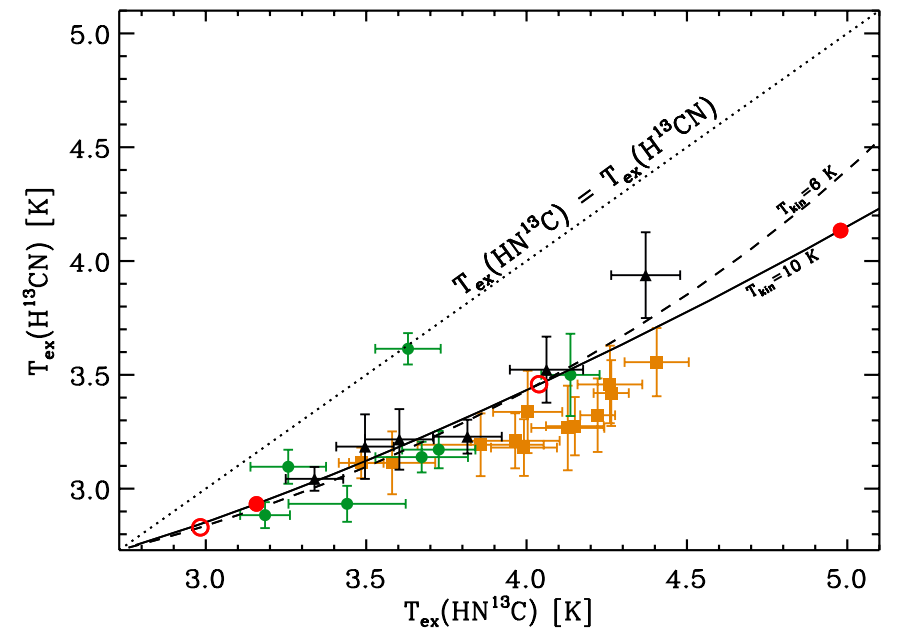

Fig. 12. Excitation temperatures of $\mathrm{H}^{13} \mathrm{CN}(1-0)$ versus $\mathrm{HN}^{13} \mathrm{C}(1-0)$ evaluated from observations of L1498 (yellow squares), L1521E (green circles), and TMC 2 (black triangles). The dashed and solid black lines trace the values for $T_{\mathrm{ex}}$ computed using RADEX for kinetic temperatures, $T_{\text {kin }}$, of 6 and $10 \mathrm{~K}$, respectively; red empty circles and red solid circles show temperatures where number density assumes values equal to $10^{4}$ and $10^{5} \mathrm{~cm}^{-3}$ for $T_{\text {kin }}=6 \mathrm{~K}$ and $10 \mathrm{~K}$, respectively. The dotted black line depicts the positions where the two excitation temperatures would be equal.

the region where $\mathrm{CO}$ is depleted (see Sect. 4), we gain information about the high density "core of the core". It is worth noting that our observations also are a test of the collisional rates for HCN and HNC (Sarrasin et al. 2010; Dumouchel et al. 2010). We illustrate this in the next section.

\subsection{Comparison of observed and expected excitation temperature}

In Fig. 12, we show the comparison between the values of the excitation temperatures of $\mathrm{H}^{13} \mathrm{CN}$ and $\mathrm{HN}^{13} \mathrm{C}$ evaluated from the simultaneous fit of the hyperfine components for the three sources examined, revealing that $T_{\mathrm{ex}}\left(\mathrm{HN}^{13} \mathrm{C}\right)$ is essentially always greater than $T_{\mathrm{ex}}\left(\mathrm{H}^{13} \mathrm{CN}\right)$ which we presume to be due to the differing collisional rates. In the same plot, two curves trace the values for $T_{\text {ex }}$ computed using RADEX (van der Tak et al. 2007) which uses the new collisional rates for HNC (Sarrasin et al. 2010; Dumouchel et al. 2010) that are no longer assumed equal to HCN. These curves assume kinetic temperatures, $T_{\text {kin }}$, equal to 6 and $10 \mathrm{~K}$, and typical values of $2 \times 10^{12} \mathrm{~cm}^{-2}$ and $0.2 \mathrm{~km} \mathrm{~s}^{-1}$ for column density and line width, respectively. We 


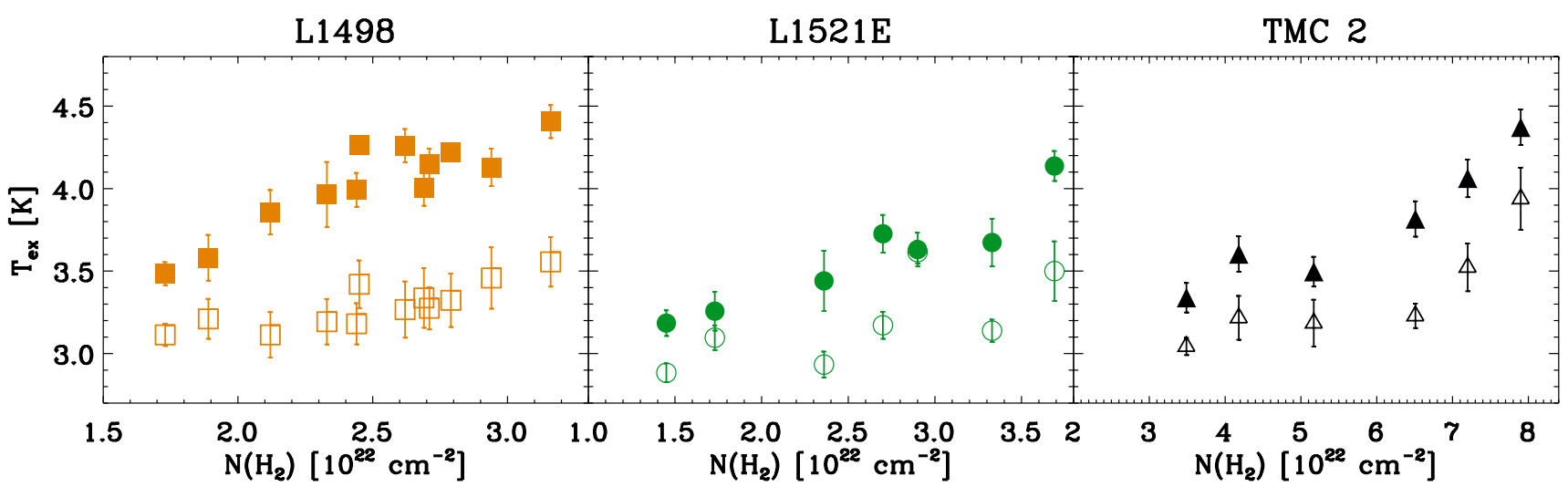

Fig. 13. Excitation temperatures of $\mathrm{H}^{13} \mathrm{CN}(1-0)$ and $\mathrm{HN}^{13} \mathrm{C}(1-0)$, empty and solid points, respectively, as a function of the molecular hydrogen column density from observations of L1498 (yellow squares), L1521E (green circles), and TMC 2 (black triangles).

also assumed that the species are cospatial and hence come from regions of the same kinetic temperature. Notice that we rely on the fact that these lines are not too optically thick and thus $T_{\text {ex }}$ determinations are relatively insensitive to column density and line width. We notice an impressive good agreement between theory and observations, even if L1498 shows values a little below the expected theoretical trend.

For L1498, we conclude from the RADEX results that the observed $T_{\mathrm{ex}}$ are consistent with a density of a few times $10^{4} \mathrm{~cm}^{-3}$ but this estimate is sensitive to the assumed temperature. If the kinetic temperature is $10 \mathrm{~K}$, then we can exclude densities as high as $10^{5} \mathrm{~cm}^{-3}$ in the region where $\mathrm{H}^{13} \mathrm{CN}$ and $\mathrm{HN}^{13} \mathrm{C}$ are undepleted. On the other hand, for a temperature of $6 \mathrm{~K}$, such densities are possible. Clearly, more sophisticated models are needed to break this degeneracy. In general, our results are consistent with the conclusion of Tafalla et al. (2006) that the HCN-HNC "hole" is smaller than that seen in CO isotopologues. This suggests that some $\mathrm{CO}$, but much less than the canonical abundance of about $10^{-4}$, is present in the $\mathrm{CO}$ depleted region to supply carbon for $\mathrm{HCN}$ and $\mathrm{HNC}$.

For L1521E and TMC 2, a central kinetic temperature of $6 \mathrm{~K}$ seems possible. From dust emission models, they are thought to reach central densities of around $3 \times 10^{5} \mathrm{~cm}^{-3}$ (Tafalla \& Santiago 2004; Crapsi et al. 2005) which would correspond to excitation temperatures for $\mathrm{H}^{13} \mathrm{CN}$ and $\mathrm{HN}^{13} \mathrm{C}$ higher than observed if the temperature was as high as $10 \mathrm{~K}$.

In Fig. 13, we show the observed excitation temperature of $\mathrm{HN}^{13} \mathrm{C}$ and $\mathrm{H}^{13} \mathrm{CN}$ against the $\mathrm{H}_{2}$ column density inferred from the dust emission. In all the three sources, one notes a clear correlation between $T_{\mathrm{ex}}$ and $N\left(\mathrm{H}_{2}\right)$. This is an important confirmation of the hypothesis that $T_{\mathrm{ex}}$ is a measure of collisional rates and that the dust emission peak is also a peak in hydrogen number density. It is also noteworthy that in TMC 2, which has the largest central column density of our three sources, the excitation temperatures continue to increase up to column densities of $8 \times 10^{22} \mathrm{~cm}^{-2}$. This suggests that $\mathrm{HN}^{13} \mathrm{C}$ and $\mathrm{H}^{13} \mathrm{CN}$ are still present in the gas phase at densities close to the maximum value in TMC $2\left(3 \times 10^{5} \mathrm{~cm}^{-3}\right.$ according to Crapsi et al. 2005). On the other hand, the $T_{\mathrm{ex}}$ values at the dust peak of TMC 2 are not greatly different from that measured at the dust peak of L1498 suggesting perhaps that the central temperature is lower in TMC 2. In any case, we conclude that excitation temperature determinations of $\mathrm{H}^{13} \mathrm{CN}$ and $\mathrm{HN}^{13} \mathrm{C}$ are a powerful technique for investigating physical parameters towards the density peaks of cores.

\subsection{Monte Carlo treatment of radiative transfer in $L 1498$}

L1498 has been already extensively studied in molecular lines (Tafalla et al. 2004, 2006; Padovani et al. 2009) and we used the Monte Carlo radiative transfer code in Tafalla et al. (2002) to model $\mathrm{H}^{13} \mathrm{CN}$ and $\mathrm{HN}^{13} \mathrm{C}$ exploiting the recent collisional rate calculations (Sarrasin et al. 2010; Dumouchel et al. 2010). The core model is the same as the one used in the analysis of the molecular survey of L1498 published in Tafalla et al. (2006). The core has a density distribution derived from the dust continuum map and an assumed constant gas temperature of $10 \mathrm{~K}$, as suggested by the ammonia analysis. The radiative transfer is solved with a slightly modified version of the Monte Carlo model from Bernes (1979). The molecular parameters were taken from the $\mathrm{LAMDA}^{2}$ database, where the rates are computed for collisions with $\mathrm{He}$ and we assumed that the $\mathrm{H}_{2}$ rates are larger than the He rates by a factor of 2, the same criterion being used for HCN in Tafalla et al. (2006).

With regard to $\mathrm{H}^{13} \mathrm{CN}$, Tafalla et al. (2006) made use of the collisional rates of Monteiro \& Stutzki (1986) which consider hyperfine structure complemented for higher transitions with those of Green \& Thaddeus (1974) for higher $J$. In this model, the abundance law is a step function with an outer value of $1.7 \times 10^{-10}$ and a central hole of $8 \times 10^{16} \mathrm{~cm}$. We ran a model with the same abundance law, but this time using the new LAMDA file for HCN, which does not account for hyperfine structure. To compensate for the lack of hyperfine structure, which spreads the photons in velocity, we broadened the line by increasing the amount of turbulence from $0.075 \mathrm{~km} \mathrm{~s}^{-1}$ to $0.2 \mathrm{~km} \mathrm{~s}^{-1}$. The upper panel of Fig. 14 shows this alternative model which nicely fits the radial profile of observed $\mathrm{H}^{13} \mathrm{CN}$ intensities and this allows us to conclude that the use of the LAMDA file for HCN without hyperfine structure has little effect on the abundance determination, the result being as good as that of Tafalla et al. (2006).

For $\mathrm{HN}^{13} \mathrm{C}$, we used the collisional rates for the main isotopologue, HNC. As for $\mathrm{H}^{13} \mathrm{CN}$, the HNC hyperfine structure is not taken into account, so we set again the parameter relative to the turbulent velocity to $0.2 \mathrm{~km} \mathrm{~s}^{-1}$. The lower panel of Fig. 14 shows the observed $\mathrm{HN}^{13} \mathrm{C}$ integrated intensities for two fits: one assumes equal abundances for $\mathrm{H}^{13} \mathrm{CN}$ and $\mathrm{HN}^{13} \mathrm{C}$, the other assumes that the $\mathrm{HN}^{13} \mathrm{C}$ abundance is 1.2 times that of $\mathrm{H}^{13} \mathrm{CN}$. These radial profiles suggest an abundance ratio between the two

${ }^{2}$ http://www.strw.leidenuniv.nl/ moldata/ 
M. Padovani et al.: Hydrogen cyanide and isocyanide in prestellar cores

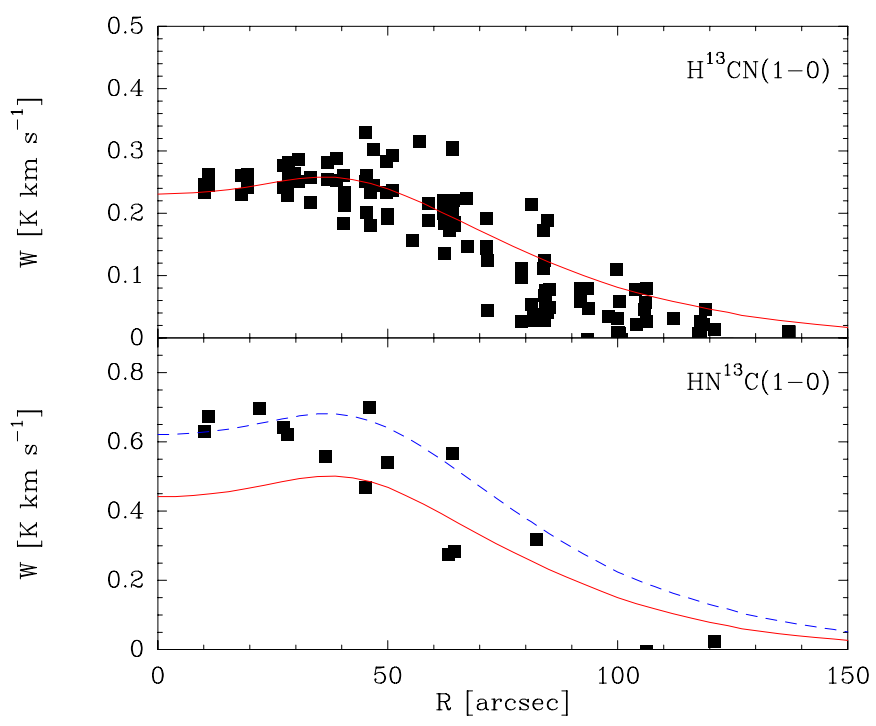

Fig. 14. Radial profile of observed $\mathrm{H}^{13} \mathrm{CN}(1-0)$ and $\mathrm{HN}^{13} \mathrm{C}(1-0)$ integrated intensities (upper and lower panel, respectively) and model prediction for a core with a central hole of $8 \times 10^{16} \mathrm{~cm}$ and an outer abundance value of $1.7 \times 10^{-10}$ (red solid lines) and $2.04 \times 10^{-10}$ (blue dashed line). $\mathrm{HN}^{13} \mathrm{C}(1-0)$ data come from this study and $\mathrm{H}^{13} \mathrm{CN}(1-0)$ data come from this study and Tafalla et al. (2006).

molecules close to 1 , with a likelihood of being closer to 1.2 towards the centre.

The Monte Carlo code also computes the radial profile of excitation temperature, which is presented in the upper panel of Fig. 15. We notice that $T_{\mathrm{ex}}$ for both species decreases with the radius from about $4.8 \mathrm{~K}$ and $3.8 \mathrm{~K}$ for $\mathrm{HN}^{13} \mathrm{C}$ and $\mathrm{H}^{13} \mathrm{CN}$, respectively, in the core interior, towards the cosmic background temperature near the outer edge of the core. Even more interesting, we found $T_{\mathrm{ex}}\left(\mathrm{HN}^{13} \mathrm{C}\right)$ systematically higher than $T_{\mathrm{ex}}\left(\mathrm{H}^{13} \mathrm{CN}\right)$, in nice agreement with the values computed from observations (see lower panel of Fig. 15 compared with Fig. 12). It is important to stress that $T_{\mathrm{ex}}$ values from observations refer to some kind of weighted mean along the line of sight for different positions, while $T_{\mathrm{ex}}$ values from Monte Carlo analysis are related to each shell in the core. This means that the two numbers are closely connected, but do not exactly have the same meaning and a proper comparison would require simulating the hyperfine analysis in the Monte Carlo spectra. However, the effect is global over the cloud and affects every layer, so it cannot be ignored in any analysis.

\section{Column density and abundance estimates}

Based on our earlier discussion, $\mathrm{HCN}$ hyperfine components are too optically thick to be used for column density determinations. This means that, while $\mathrm{H}^{13} \mathrm{CN}$, as well as $\mathrm{HN}^{13} \mathrm{C}$, originate in the central part of the core, $\mathrm{HCN}$ is dominated by the foreground layer emission and hence it is not possible to observe the centre of the core. Besides, there is an unequivocal difference between the $\mathrm{H}^{13} \mathrm{CN}(F=2 \rightarrow 1)$ and the $\operatorname{HCN}(F=0 \rightarrow 1)$ profiles (see Fig. 9). In fact, $\mathrm{H}^{13} \mathrm{CN}$ seems to be much more Gaussian than the weakest HCN line and it is likely to underestimate the optical depth of HCN.

However, we found that even $\mathrm{HN}^{13} \mathrm{C}$ and $\mathrm{H}^{13} \mathrm{CN}$ are moderately optically thick (see Sect. 3 ) and in this case one can derive the column density in the $j$ th level, $N_{j}$, integrating Eq. (2) over
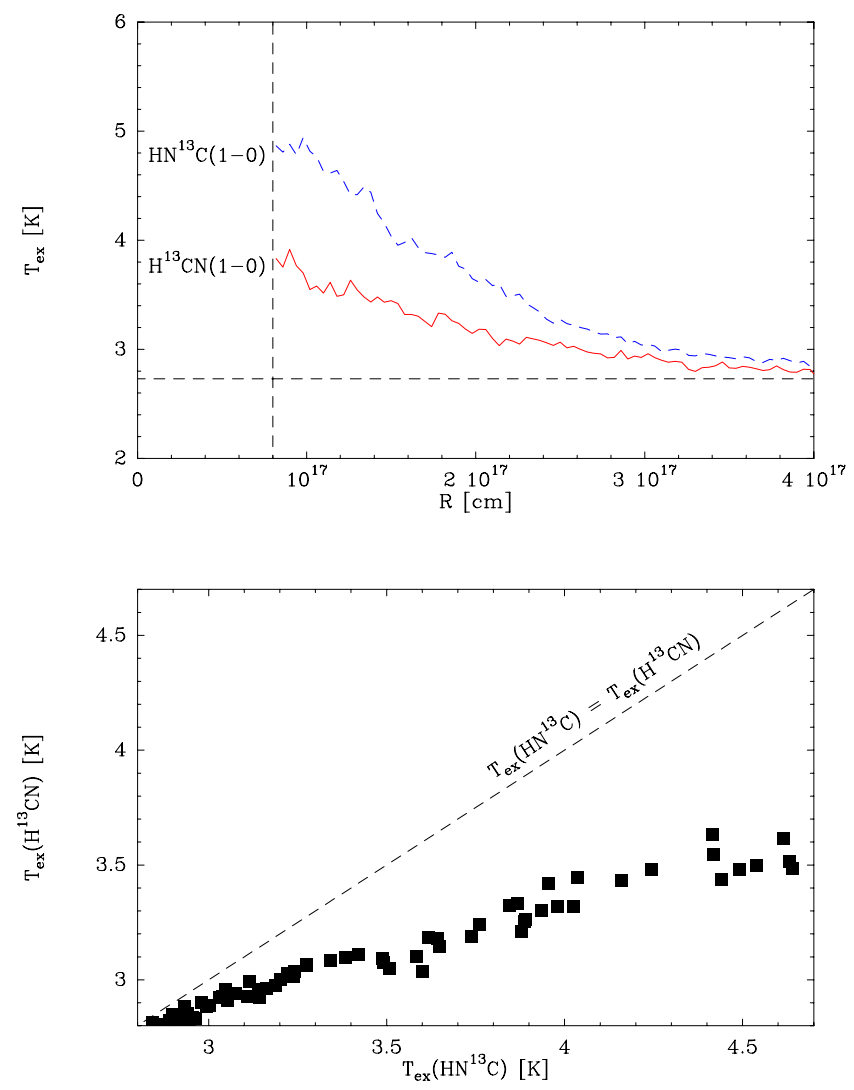

Fig. 15. Upper panel: radial profile of excitation temperature for $\mathrm{H}^{13} \mathrm{CN}(1-0)$, red solid line, and $\mathrm{HN}^{13} \mathrm{C}(1-0)$, blue dashed line, in L1498 as predicted by our best fit Monte Carlo model; the vertical dashed line represents the central hole radius and the horizontal dashed line shows the cosmic background temperature limit. Lower panel: comparison between $\mathrm{HN}^{13} \mathrm{C}(1-0)$ and $\mathrm{H}^{13} \mathrm{CN}(1-0)$ local values of the excitation temperature in each core shell; the dashed line depicts the positions where the two excitation temperatures would be equal.

frequency to obtain the integrated intensity, $W_{j}$, with the optical depth given by

$\tau=\frac{c^{3}}{8 \pi v_{j i}^{3}} A_{j i} N_{j}\left(\mathrm{e}^{h v_{j i} / k T_{\mathrm{ex}}}-1\right) \phi(v)$,

where $A_{j i}$ is the Einstein coefficient, and $\phi(v)$ the profile function, which is a sum of Gaussians (assuming a Maxwellian distribution of the particle velocities) with the appropriate weights and shifts with respect to the central frequency, properly accounting for the hyperfine structure. As shown in Fig. 16, for small optical depths $(\tau<1) N_{j}$ is in direct ratio to $W_{j}$ while, as $\tau$ increases, the curve flattens even if the flattening is not very sharp because when the main component is thick, the satellites are still thin. Allowing for increasing optical depth is important not to underestimate column density: as an instance, at the emission peak of $\mathrm{H}^{13} \mathrm{CN}$ in L1498, where $\tau=4.58$ (see Sect. 3), the linear approximation causes an inaccuracy of about $45 \%$ and this percentage mainly depends on the optical depth, but not on $T_{\text {ex }}$. In fact, for decreasing excitation temperatures, the linear approximation decreases its slope, but also the flattening due to opacity is accentuated. $\mathrm{HN}^{13} \mathrm{C}$ indicates a similar deviation.

Hence, we determine the column density $N_{j}$ corresponding to the observed $W_{j}$ carrying out this procedure using the maximum and the minimum value of the excitation temperature in order to estimate errors in $N_{j}$. It is important to remark that in 


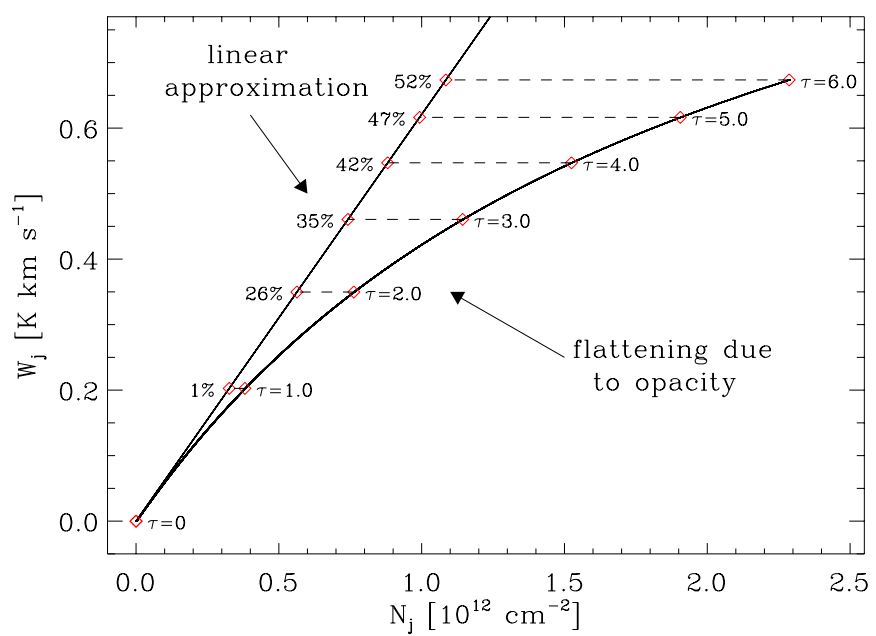

Fig. 16. $\mathrm{H}^{13} \mathrm{CN}(1-0)$ integrated intensity as a function of the column density of the $J=1$ level for $T_{\mathrm{ex}}=3.56 \mathrm{~K}$ (the highest excitation temperature observed in L1498). The percentage values show the deviation of the linear approximation with respect to the correct value taking account for optical depth.

this way column densities are directly estimated from the integrated intensity of the spectra, avoiding the use of optical depth values evaluated from the fit of the hyperfine components which have often large uncertainties (see Fig. 4). Finally, for the total column density, $N$, it holds

$\frac{N_{j}}{N}=\frac{g_{j}}{Q} \mathrm{e}^{-E_{j} / k T_{\text {ex }}}$,

where $Q=\sum_{j=0}^{\infty} g_{j} \mathrm{e}^{-E_{j} / k T_{\text {ex }}}$ is the partition function, $E_{j}$ and $g_{j}$ being the energy and the statistical weight of the upper $j$ th level, respectively.

Hence, we derive the column density and the abundance of these species with respect to molecular hydrogen for the main isotopologues $\mathrm{HNC}$ and $\mathrm{HCN}$, assuming the canonical isotopic $\left[{ }^{12} \mathrm{C}\right] /\left[{ }^{13} \mathrm{C}\right]$ ratio (Milam et al. 2005). Figure 17 shows HNC and $\mathrm{HCN}$ column densities as a function of $\mathrm{H}_{2}$ column density which has been calculated from the millimetre dust emission, assuming a dust opacity per unit mass of $0.005 \mathrm{~cm}^{2} \mathrm{~g}^{-1}$ and a dust temperature of $10 \mathrm{~K}$. Notice the correlation between HNC and HCN column densities and their optical depths in Fig. 4 for L1498: for $N\left(\mathrm{H}_{2}\right) \gtrsim 2.5 \times 10^{22} \mathrm{~cm}^{-2}, N(\mathrm{HNC})$ becomes higher than $N(\mathrm{HCN})$ as well as $\tau(\mathrm{HNC})>\tau(\mathrm{HCN})$. Besides, $\mathrm{HNC}$ and $\mathrm{HCN}$ abundances increase towards the peak in TMC 2 but not in the other two cores.

As an instance, in Table 1 we show the values of column density and abundance with respect to $\mathrm{H}_{2}$ for $\mathrm{HNC}$ and $\mathrm{HCN}$ at the dust emission peak.

From the point of view of the chemistry, it is even more interesting to check the ratio of abundances $[\mathrm{HNC}] /[\mathrm{HCN}]$ as a function of the column density of molecular hydrogen. Results for the three sources of our sample are showed in Fig. 18. We found a rather constant value for this ratio, with weighted mean values of $0.94 \pm 0.11$ for L1498, $1.14 \pm 0.11$ for L1521E, and $1.05 \pm 0.10$ for TMC 2, confirming the result achieved by Sarrasin et al. (2010) and Dumouchel et al. (2010), and consistent with detailed chemical models available in the literature (e.g. Herbst et al. 2000).

This result resolves an important discrepancy between theory and observations which has lasted almost twenty years. There are rather few clear predictions of chemistry theory, but we can
Table 1. Column densities and abundances for $\mathrm{HNC}$ and $\mathrm{HCN}$ at the dust emission peak in our source sample.

\begin{tabular}{lcc}
\hline \hline source & $\begin{array}{c}N(\mathrm{HNC}) \\
{\left[10^{14} \mathrm{~cm}^{-2}\right]}\end{array}$ & $\begin{array}{c}N(\mathrm{HCN}) \\
{\left[10^{14} \mathrm{~cm}^{-2}\right]}\end{array}$ \\
\hline L1498 & $1.58(0.56)$ & $1.24(0.18)$ \\
L1521E & $0.51(0.14)$ & $0.47(0.12)$ \\
TMC 2 & $2.41(0.42)$ & $1.86(0.26)$ \\
\hline & {$[\mathrm{HNC}] /\left[\mathrm{H}_{2}\right]$} & {$[\mathrm{HCN}] /\left[\mathrm{H}_{2}\right]$} \\
& {$\left[10^{-9}\right]$} & {$\left[10^{-9}\right]$} \\
\hline L1498 & $5.00(1.95)$ & $3.92(0.96)$ \\
L1521E & $1.36(0.42)$ & $1.27(0.37)$ \\
TMC 2 & $3.05(0.57)$ & $2.35(0.36)$ \\
\hline
\end{tabular}

confirm that HNC and HCN seem to have similar abundances. However, given that what we measure is the column density of isotopologues, this implies that any fractionation should be the same for the two of them.

Observations of higher-level transitions of $\mathrm{HN}^{13} \mathrm{C}$ and $\mathrm{H}^{13} \mathrm{CN}$ as well as $\mathrm{H}^{15} \mathrm{NC}$ and $\mathrm{HC}^{15} \mathrm{~N}$ would help to refine the determination of column densities and would allow to estimate the isotopic $\left[{ }^{14} \mathrm{~N}\right] /\left[{ }^{15} \mathrm{~N}\right]$ ratio, too.

\section{Conclusions}

We have studied in this article the behaviour of the $J=1 \rightarrow 0$ transitions of $\mathrm{HCN}(1-0), \mathrm{H}^{13} \mathrm{CN}(1-0)$, and $\mathrm{HN}^{13} \mathrm{C}(1-0)$ as a function of position in the three starless cores L1498, L1521E, and TMC 2. We also observed $\mathrm{N}_{2} \mathrm{H}^{+}(1-0)$ and $\mathrm{C}^{18} \mathrm{O}(2-1)$ in TMC 2. Our main conclusions are as follows.

1. $\mathrm{H}^{13} \mathrm{CN}(1-0)$ and $\mathrm{HN}^{13} \mathrm{C}(1-0)$ are often assumed to be optically thin when computing column densities. Our results show that in the sources studied by us, this is inaccurate and indeed, optical depths are sufficiently high to make a reasonable estimate of the excitation temperatures of these transitions which can be compared with model predictions.

2. The plot of $\mathrm{H}^{13} \mathrm{CN}(1-0)$ excitation temperature against $\mathrm{HN}^{13} \mathrm{C}(1-0)$ excitation temperature follows the curve expected based on the collision rates recently computed by Sarrasin et al. (2010) and Dumouchel et al. (2010) thus confirming these results. This plot also stresses the importance of calculations of potential surfaces and collisional coefficients for isotopologues separately. Moreover these excitation temperatures correlate well with $\mathrm{H}_{2}$ column density estimated on the basis of dust emission showing that dust emission peaks really do trace peaks in the gas density.

3. These latter results combined with our intensity-offset plots demonstrate convincingly that, at least in TMC 2, HCN and HNC survive in the gas phase at densities above $10^{5} \mathrm{~cm}^{-3}$ where $\mathrm{CO}$ has depleted out. The implication of this is likely that $\mathrm{CO}$ survives at abundances of a few percent of its canonical value of around $10^{-4}$ and supplies the carbon required in lower abundance species.

4. The profiles of the three satellites of $\mathrm{HCN}(1-0)$ become increasingly "skew" with increasing optical depth and indeed the corresponding $\mathrm{H}^{13} \mathrm{CN}(1-0)$ profiles are reasonably symmetric. This behaviour suggests the possibility of modelling the velocity field and abundance along the line of sight.

5. We have used a model of the density distribution in L1498 to describe the $\mathrm{HN}^{13} \mathrm{C}(1-0)$ and $\mathrm{H}^{13} \mathrm{CN}(1-0)$ results and find reasonable agreement with a model based on the previous observations of Tafalla et al. (2006) containing a central "depletion hole" of radius $8 \times 10^{16} \mathrm{~cm}$. This does not 


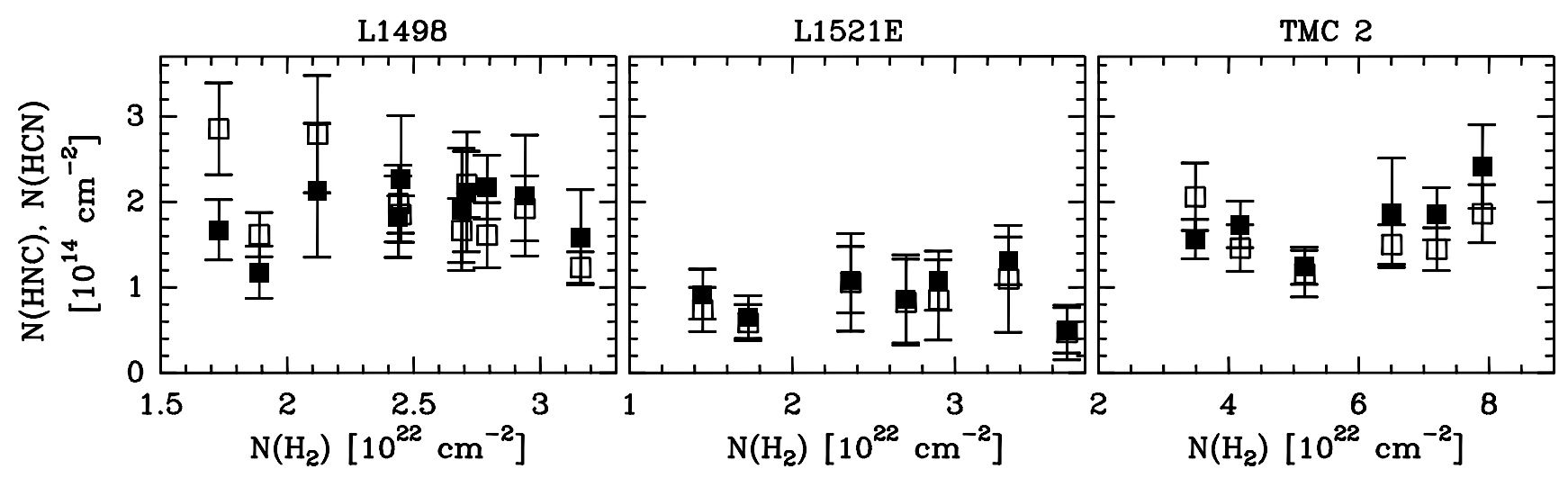

Fig. 17. Column density of $\mathrm{HNC}(1-0)$, solid squares, and $\mathrm{HCN}(1-0)$, empty squares for the source sample as a function of the $\mathrm{H}_{2}$ column density.

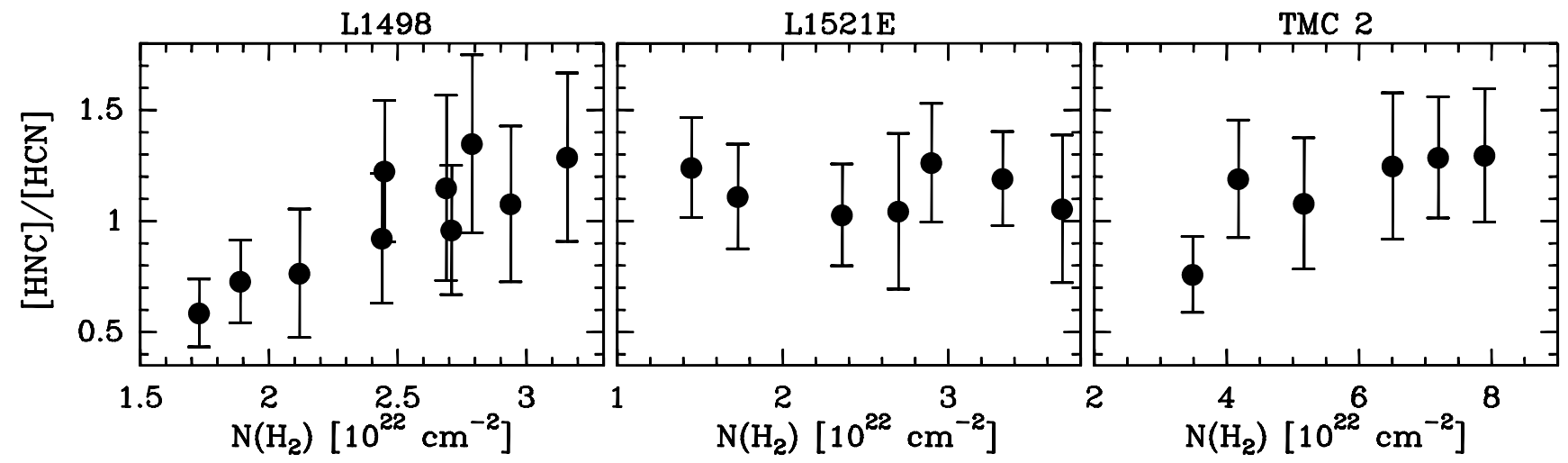

Fig. 18. Abundance ratio $[\mathrm{HNC}] /[\mathrm{HCN}]$ for the source sample as a function of the $\mathrm{H}_{2}$ column density.

exclude models without a depletion hole, but does confirm our conclusions on the excitation discussed above. Indeed, rather surprisingly, our results suggest that $\mathrm{HN}^{13} \mathrm{C}(1-0)$ and $\mathrm{H}^{13} \mathrm{CN}(1-0)$ trace the high density nuclei of these cores when compared with other carbon-bearing species.

6. Our results are consistent with the models of Herbst et al. (2000) who found that HNC and HCN should have similar abundances in prestellar cores.

Acknowledgements. This work has benefited from research funding from the European Community's Seventh Framework Programme. We thank the anonymous referee for her/his very interesting comments that helped to improve the paper.

\section{Appendix A: Non-LTE hyperfine populations}

For a homogeneous slab with LTE between different hyperfine levels, one has

$R_{i j}=\frac{1-\exp \left(-f_{i} \tau\right)}{1-\exp \left(-f_{j} \tau\right)}$

where $\tau$ is the total transition optical depth and $f_{i}$ is the relative line strength of the $i$ th component as in Table B.1. We applied this procedure to all the transitions, except for $\mathrm{HN}^{13} \mathrm{C}(1-0)$, because of the blending of the hyperfine components. Using the same method adopted in Padovani et al. (2009) for $\mathrm{C}_{2} \mathrm{H}$, we consider a homogeneous slab, then a two-layer model, and we compare different couples of ratios one versus the other to quantify the possible departure from LTE. As for $\mathrm{C}_{2} \mathrm{H}$, we conclude that also a two-layer model cannot explain the observed intensities, requiring a proper non-LTE treatment.
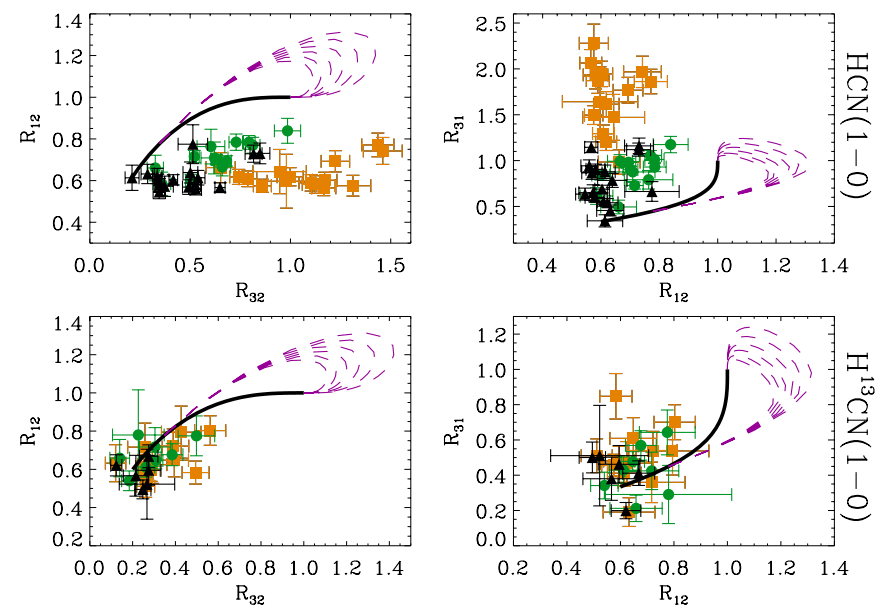

Fig. A.1. Ratio of the integrated intensities of couples of components of $\mathrm{HCN}(1-0)$ (upper panels), and $\mathrm{H}^{13} \mathrm{CN}(1-0)$ (lower panels), where $R_{i j}$ represents the ratio between the integrated intensities $W_{i}$ and $W_{j}$ (see Table B.1 for component indices). Observational data: L1498 (yellow squares), L1521E (green circles), and TMC 2 (black triangles). One-layer model (black solid curve), two-layer model (magenta dashed curves).

Comparing the upper and the lower panels of Fig. A.1 related to $\mathrm{HCN}(1-0)$ and $\mathrm{H}^{13} \mathrm{CN}(1-0)$, respectively, it is clear that real departures from LTE are present and are stronger in L1498 than in L1521E and TMC 2. In L1498, $R_{32}$, the ratio between the weakest $(88633 \mathrm{MHz})$ and the strongest $(88631 \mathrm{MHz})$ component, is larger than unity and $R_{31}>1$ in all the positions. This suggests a very high self (or foreground) absorption of the 
strongest component, as found for the $\mathrm{C}_{2} \mathrm{H}(1-0)$ emission in the same core (Padovani et al. 2009). The other two cores show ratios which deviate from the LTE curve revealing again the presence of optical depth effects. As an instance, in the optically thin limit, $R_{32}$ should be equal to about 0.2 , but the arithmetic mean of $R_{32}$ is $\sim 0.60$ for L1521E and $\sim 0.47$ for TMC 2 .

Finally, an important remark follows from the $\mathrm{H}^{13} \mathrm{CN}(1-0)$ ratios (lower panels): minor deviations from LTE are present even in this less abundant isotopologue which should be more optically thin. Even if optically thick lines are the foremost responsible for the arising of non-LTE effects, the density can be high enough to allow transition within the same rotational level (e.g. the transition $J, F=1,2 \rightarrow 1,1$ has the same order of magnitude of rates relative to a transition between different rotational levels, see Monteiro \& Stutzki 1986).

\section{Appendix B: Spectroscopic data and observational parameters}

Table B.1. $\mathrm{HCN}(1-0)$ and $\mathrm{H}^{13} \mathrm{CN}(1-0)$ frequencies of the hyperfine components (From the JPL Molecular Spectroscopy Database: http://spec.jpl.nasa.gov).

\begin{tabular}{lccc}
\hline \hline Comp No. & $F^{\prime}-F$ & Frequency $[\mathrm{MHz}]$ & $f$ \\
\hline \multicolumn{4}{c}{$\mathrm{HCN}(1-0)$} \\
1 & $1-1$ & 88630.4160 & 0.333 \\
2 & $2-1$ & 88631.8470 & 0.556 \\
3 & $0-1$ & 88633.9360 & 0.111 \\
\hline \multicolumn{4}{c}{$\mathrm{H}^{13} \mathrm{CN}(1-0)$} \\
1 & $1-1$ & 86338.7670 & 0.333 \\
2 & $2-1$ & 86340.1840 & 0.556 \\
3 & $0-1$ & 86342.2740 & 0.111 \\
\hline
\end{tabular}

Table B.2. $\mathrm{HN}^{13} \mathrm{C}(1-0)$ frequencies of the hyperfine components.

\begin{tabular}{lccc}
\hline \hline Comp No. & transition & Frequency $[\mathrm{MHz}]$ & $f$ \\
\hline \multicolumn{4}{c}{$\mathrm{HN}^{13} \mathrm{C}(1-0)$} \\
1 & $F_{1}^{\prime}-F_{1}=0-1$ & 87090.675 & 0.065 \\
2 & $F_{2}^{\prime}=2$ & 87090.791 & 0.264 \\
3 & $F_{2}^{\prime}=3$ & 87090.834 & 0.432 \\
4 & $F_{1}^{\prime}-F_{1}=1-1$ & 87090.886 & 0.239 \\
\hline
\end{tabular}

Notes. For a complete list of the hyperfine components, see van Der Tak et al. (2009). In fact, there are four overlapped hyperfine components with $F_{2}^{\prime}=2$, three components with $F_{2}^{\prime}=3$, and three components with $F=1 \rightarrow 1$.
Table B.3. Summary of observed molecules together with the observing parameters: half power beamwidth, beam and forward efficiencies, system temperature, and precipitable water vapor.

\begin{tabular}{lccccc}
\hline \hline transition & $\begin{array}{c}H P B W \\
{\left[{ }^{\prime \prime}\right]}\end{array}$ & $B_{\text {eff }}$ & $F_{\text {eff }}$ & $\begin{array}{c}T_{\text {sys }} \\
{[\mathrm{K}]}\end{array}$ & $\begin{array}{c}\text { pwv } \\
{[\mathrm{mm}]}\end{array}$ \\
\hline $\mathrm{HCN}(1-0)$ & 28 & 0.77 & 0.95 & $\sim 130$ & $1-2$ \\
$\mathrm{H}^{13} \mathrm{CN}(1-0)$ & 28 & 0.77 & 0.95 & $\sim 120$ & $1-2$ \\
$\mathrm{HN}^{13} \mathrm{C}(1-0)$ & 28 & 0.77 & 0.95 & $\sim 140$ & $1-2$ \\
$\mathrm{~N}_{2} \mathrm{H}^{+}(1-0)$ & 26 & 0.77 & 0.95 & $\sim 160$ & $1-2$ \\
$\mathrm{C}^{18} \mathrm{O}(2-1)$ & 11 & 0.55 & 0.91 & $\sim 320$ & $1-2$ \\
\hline
\end{tabular}

\section{References}

Akyilmaz, M., Flower, D. R., Hily-Blant, P., Pineau Des Forêts, G., \& Walmsley, C. M. 2007, A\&A, 462, 221

Bacmann, A., Lefloch, B., Ceccarelli, C., et al. 2002, A\&A, 389, L6

Bergin, E. A., \& Tafalla, M. 2007, ARA\&A, 45, 339

Bernes, C. 1979, A\&A, 73, 67

Bisschop, S. E., Fraser, H. J., Öberg, K. I., van Dishoeck, E. F. \& Schlemmer, S. 2006, A\&A, 449, 1297

Brady Ford, A., \& Shirley, Y. L. 2011, ApJ, 728, 144

Caselli, P., Walmsley, C. M., Zucconi, A., et al. 2002a, ApJ, 565, 344

Caselli, P., Benson, P. J., Myers, P. C., \& Tafalla, M. 2002b, ApJ, 572, 238

Crapsi, A., Caselli, P., Walmsley, C. M., et al. 2005, ApJ, 619, 379

Dumouchel, F., Faure, A., \& Lique, F. 2010, MNRAS, 406, 2488

Flower, D. R., Pineau Des Forêts, G., \& Walmsley, C. M. 2006, A\&A, 456, 215

Green, S., \& Thaddeus, P. 1974, ApJ, 191, 653

Herbst, E., Terzieva, R., \& Talbi, D. 2000, MNRAS, 311, 869

Hily-Blant, P., Walmsley, C. M., Pineau des Forêts, G., \& Flower, D. 2008, A\&A, 480, L5

Hily-Blant, P., Walmsley, C. M., Pineau des Forêts, G., \& Flower, D. 2010, A\&A, 513, A41 (Paper I)

Mardones, D., Myers, P. C., Tafalla, M., et al. 1997, ApJ, 489, 719

Milam, S. N., Savage, C., Brewster, M. A., Ziurys, L. M., \& Wyckoff, S. 2005, ApJ, 634, 1126

Monteiro, T. S., \& Stutzki, J. 1986, MNRAS, 221, P33

Padovani, M., Walmsley, C. M., Tafalla, M., Galli, D., \& Müller, H. S. P. 2009, A\&A, 505, 1199

Pagani, L., Daniel, F., \& Dubernet, M.-L. 2009, A\&A, 494, 719

Pontoppidan, K. M. 2006, A\&A, 453, L47

Sarrasin, E., Abdallah, D. B., Wernli, M., et al. 2010, MNRAS, 404, 518

Schmid-Burgk, J., Muders, D., Müller, H. S. P., \& Brupbacher-Gatehouse, B. 2004, A\&A, 419, 949

Tafalla, M., \& Santiago, J. 2004, A\&A, 414, L53

Tafalla, M., Myers, P. C., Caselli, P., Walmsley, C. M., \& Comito, C. 2002, ApJ, 569,815

Tafalla, M., Myers, P. C., Caselli, P., \& Walmsley, C. M. 2004, A\&A, 416, 191

Tafalla, M., Santiago-García, J., Myers, P. C., et al. 2006, A\&A, 455, 577

van der Tak, F. F. S., Black, J. H., Schöier, F. L., Jansen, D. J., \& van Dishoeck, E. F. 2007, A\&A, 468, 627

van der Tak, F. F. S., Müller, H. S. P., Harding, M. E., \& Gauss, J. 2009, A\&A, 507,347

Walmsley, C. M., Churchwell, E., Nash, A., \& Fitzpatrick, E. 1982, ApJ, 258, L75 